K. TOHGE

KODAI MATH. J.

11 (1988), 249-279

\title{
MEROMORPHIC FUNCTIONS COVERING CERTAIN FINITE SETS AT THE SAME POINTS
}

\author{
By KazUYA TOHgE
}

\section{Introduction.}

We say that meromorphic functions $f$ and $g$ share a value $c$ provided that $f(z)=c$ if and only if $g(z)=c$ (regardless of multiplicities). When we need to consider their multiplicities, we shall make use of the abbreviation $C M$, following Gundersen [4]. Unless stated otherwise, all functions will be assumed to be nonconstant and meromorphic in the plane. It is also assumed that the reader is familiar with usual notations of Nevanlinna theory of meromorphic functions (see, for example, [5]).

Our main interest in this paper lies in the following question: under what circumstances two different functions share the values? $R$. Nevanlinna $[8,9]$ proved that if two functions share five distinct values (possibly including $\infty$ ), then they must be identical. The functions $\exp (z)$ and $\exp (-z)$, with the values $0,1,-1, \infty$, show that here 5 cannot replaced by 4 . He has also shown that three or four values are, apart from certain exceptional cases, sufficient to determine a function $f(z)$, if we know in addition the multiplicity of the roots of the equation $f(z)=c$. For entire functions, their relationships have been given in the various specific circumstances. Here we prove some corresponding results for meromorphic functions in the plane.

\section{Statement and discussion of results.}

Let $\left\{a_{n}\right\},\left\{b_{n}\right\}$ and $\left\{p_{n}\right\}$ be three disjoint sequences with no finite limit points. If there is a meromorphic function $f$ whose zeros, $d$-points and poles are exactly $\left\{a_{n}\right\},\left\{b_{n}\right\}$ and $\left\{p_{n}\right\}$ respectively, then the given triple $\left(\left\{a_{n}\right\},\left\{b_{n}\right\}\right.$, $\left.\left\{p_{n}\right\}\right)$ is called the zero-d-pole set. Here of course $d$ is a nonzero complex number. If further there exists only one such function $f$, then the triad is said to be unique. Unicity in this sense does not hold in general, and we have the following theorem.

THEOREM 1. Let $\left(\left\{a_{n}\right\},\left\{b_{n}\right\},\left\{p_{n}\right\}\right)$ and $\left(\left\{a_{n}\right\},\left\{c_{n}\right\},\left\{p_{n}\right\}\right)$ be the zero-onepole set and the zero-d-pole set of a function $N$, where $d \neq 1$. Then at least one of two given triads is unique, unless $N$ is one of the following forms;

Received December 21, 1987 


$$
\left(e^{L(z)}+A\right)^{ \pm 1} \text { and }\left(\frac{e^{L(z)}-d}{e^{L(z)}-1}\right)^{ \pm 1},
$$

where $A$ is an arbitrary constant and $L$ is an entire function.

The result for entire $N$ has been proved by Ozawa [11]. We see easily that if $N$ is of the latter forms, then another function, whose zero-one-pole set or zero- $d$-pole set is just the given triad in this theorem, is either $N / d$ or $d N$. The more reasonable assumptions are made about $N$, the more we can say about unicity of its zero- $d$-pole sets.

Examples. The zero- $d$-pole set of $\sin (z)$ is unique for any nonzero complex number $d$. That of $\tan (z)$ is also unique if $d \neq \pm i$, and for $d= \pm i$ it is common to $-\tan (z)$.

The former was given by Rubel and Yang [12] with $d=1$, and for the latter we observe $i \cdot \tan (z)=\{\exp (2 i z)-1\} /\{\exp (2 i z)+1\}$. These results are shown by the similar method to Theorem 1 .

Now we shall confine the values shared by functions to $0,1, \infty$. It is, however, not sufficient in general to determine their relationships. Therefore we shall impose the following additional assumption:

THEOREM 2. Let $f(z)$ and $g(z)$ be two transcendental functions. Suppose that they share $0,1, \infty C M$ and their first derivatives share a finite valve a CM. If (A) $a=0$, or (B) $a \neq 0$ and further

$\operatorname{Max}\left[\limsup \log \log T(r, f) / \log r, \limsup _{r \rightarrow \infty} \log \log T(r, g) / \log r\right]<1$, then they must satisfy exactly one of the following relations;

$$
\begin{aligned}
& f=g, \quad f \cdot g=1, \quad(f-1)(g-1)=1, \quad \text { and } \\
& \{(C-1) f-C\}\{(C-1) g+1\}=-C,
\end{aligned}
$$

in addition to $(A)$,

$$
f=C g, \quad f+g=1, \quad f-1=C(g-1),
$$

with a constant $C \neq 0,1$. Here for $(B) f$ and $g$ must be entire of order exactly one, unless $f=g$.

These assumptions of $C M$ have the necessity in some extent. In fact, for example, C.C. Yang [13] proved the following result for entire functions;

THEOREM. Let $f(z)$ and $g(z)$ be two transcendental entire functions satisfying (a) they share $0 C M$ and all zeros are simple, (b) their first derivatives share 0 $C M$, and 
(c) $\operatorname{Max}\left[\limsup \log \log \log M(r, f) / \log r, \limsup _{r \rightarrow \infty} \log \log \log M(r, g) / \log r\right]<1$.

Then they satisfy exactly one of the following two relations:

(I) $f(z)=C\{g(z)\}^{k}$ where $C$ and $k$ are constants, or

(II) $f(z)=C_{1} \exp \{\gamma(z)\}+C_{2}, g(z)=C_{3}\left[C_{2} \exp \{-\gamma(z)\}+C_{1}\right]$ where $C_{1}, C_{2}$ and $C_{3}$ are constants and $\gamma$ is entire of order less than one.

Here we suppose further that $f$ and $g$ share 1 with no consideration of multiplicity, then the simple analysis implies that they share $1 C M$ (so we regard if they assume no 1-point). Also his example $\exp (2 z)$ and $\exp \left(e^{z}\right)$ show that Condition (c) is necessary, however in our theorem, a question whether the restrictions on $C M$ and on their hyper-orders in $(B)$ can be relaxed still remains to be answered. It will be seen in the proof the actual representations for the pair of functions to satisfy the above relationships.

Following the study of Gross [1], we shall consider the functions $f$ and $g$ covering certain finite sets (possibly including $\infty$ ), instead of certain values, at the same points. In other words, we observe the circumstances that $f(z) \in S$ if and only if $g(z) \in S$ for some sets $S$. For the purpose of convenience we shall make common use of the abbreviation $C M$ also in this case. Now we choose the following three sets $S_{1}=\{-1,1\}, S_{2}=\{0\}$ and $S_{3}=\{\infty\}$ or more generally;

Theorem 3. Let $n$ be an integer $(\geqq 2)$ and $S_{1}=\left\{\zeta \in C: \zeta^{n}=1\right\}, S_{2}=\{0\}$ and $S_{3}=\{\infty\}$. Suppose that two functions $f$ and $g$ satisfy

$$
f(z) \in S_{i} \text { if and only if } g(z) \in S_{i} C M, \quad i=1,2,3 .
$$

Then they must satisfy either of the following two relations;

(a) $f(z)=C g(z)$, where $C$ is a constant with $C^{n}=1$, or

(b) $f(z)^{n}=\exp \{\alpha(z)\}$ and $g(z)^{n}=\exp \{-\alpha(z)\}$ with an entire function $\alpha$, that is, $f(z) \cdot g(z)=C_{1}$ for a constant $C_{1}$ with $C_{1}^{n}=1$.

Gross and Osgood have proven this result in [2] for entire $f$ and $g$ of finite order with $n=2$. This theorem shows that the next two are only possible pairs of functions, which share four values $-1,0,1, \infty C M$, excepting $f=g$ :

$$
f(z)=e^{\alpha(z)}, \quad g(z)=e^{-\alpha(z)}
$$

and

$$
f(z)=-g(z)=\frac{\exp \{\alpha(z)\}-1}{\exp \{\alpha(z)\}+1}
$$

with an entire function $\alpha$.

In this context, we may also consider the sats $S_{1}=\{1\}, S_{2}=\{-1\}, S_{3}=$ $\{0, a\}$ and $S_{4}=\{\infty\}$ with $a \neq 0, \pm 1, \infty$, and prove

THEorem 4. Let $S_{1}=\{1\}, S_{2}=\{-1\}, S_{3}=\{a, b\}$ and $S_{4}=\{\infty\}$ with $S_{i} \cdot S_{j}=\phi$ $(i \neq j)$. Suppose that two functions $f$ and $g$ satisfy 


$$
f \in S_{i} \text { if and only if } g \in S_{i} C M, \quad i=1, \cdots, 4 .
$$

Then they must be connected with exactly one of the following relations;

$$
\begin{aligned}
& f= \pm g, \quad f \cdot g=1, \quad f+g= \pm 2, \quad(f \pm 1)(g \pm 1)=4, \\
& f \pm 1=\omega(g \pm 1), \quad \text { and }\left(f+\frac{1+\omega}{1-\omega}\right)\left(g-\frac{1+\omega}{1-\omega}\right)=\frac{4}{3}
\end{aligned}
$$

where $\omega$ is a cubic root of unity different from 1 and double signs in same order respectively. Further if all of $a=-b, a \cdot b=1$ and $(1 \pm a)(1 \pm b)=4$ fail, then $f=g$.

The corresponding result for entire functions was obtained by Gross [1] and there were introduced only three relations, $f=g, f \cdot g=1$ and $(f-1)(g-1)=4$. However additional relations $(f+1)(g+1)=4$ and $(f+c)(g-c)=4 / 3$ with $c=$ $(1+\omega) /(1-\omega)$ should have been done. The concrete representations for $f$ and $g$ are given in our proof, and these give the same as Theorem 3 for $a=b=0$.

Also the sets of two elements were considered by Gross and Yang [3], assuming two functions to be entire and of finite order. In this connection, we are led to the following result by the idea of Theorem 1 which is closely related to so-called Nevanlinna's three-function theorem $[8,9]$.

THEOREM 5. Let $S_{1}=\left\{a_{1}, a_{2}\right\}, S_{2}=\left\{b_{1}, b_{2}\right\}$ and $S_{3}=\left\{c_{1}, c_{2}\right\}$ be three pairs of elements whose sum is equal to $d \in C$ but multiplications are different from one another. Suppose that there exist three functions $f, g$ and $h$ such that;

$f \in S_{i}$ if and only if $g \in S_{i} C M, i=1,2$, while

$f \in S_{j}$ is and only if $h \in S_{j} C M, j=1,3$, and further

$f, g$ and $h$ share $\infty C M$.

Then either ( I ) one of $f=g, f+g=d, f=h$ and $f+h=d$, or else

(II) $g=h$, or $g+h=d$, and $(f-(1 / 2) d)(g-(1 / 2) d)= \pm\left(a_{1} a_{2}-c_{1} c_{2}\right)$ with $c_{1}=c_{2}$ and $a_{1} a_{2}-b_{1} b_{2}=2\left(a_{1} a_{2}-c_{1} c_{2}\right)$,

(III) $g=h$, or $g+h=d$, and $(f-(1 / 2) d)(g-(1 / 2) d)= \pm\left(a_{1} a_{2}-b_{1} b_{2}\right)$ with $b_{1}=b_{2}$ and $a_{1} a_{2}-c_{1} c_{2}=2\left(a_{1} a_{2}-b_{1} b_{2}\right)$,

(IV) $(f-(1 / 2) d)(g-(1 / 2) d)= \pm(f-(1 / 2) d)(h-(1 / 2) d) / K= \pm\left(a_{1} a_{2}-b_{1} b_{2}\right)$ with $a_{1}=a_{2}$ and $a_{1} a_{2}-c_{1} c_{2}=K\left(a_{1} a_{2}-b_{1} b_{2}\right), K \in \boldsymbol{C}-\{0,1\}$.

In the proof we see certain forms of these functions which are related as mentioned above. As an immediate consequence of this theorem, we see that if all the $S_{i}(i=1,2,3)$ have distinct elements then ( $\left.\mathrm{I}\right)$ is only the possible case.

\section{Lemmas.}

All our proofs depend on the impossibility of Borel's identity [9], which is expressed as follows: 
LEMMA $1[10]$. Let $\left\{\alpha_{j}\right\}$ be a set of nonzero constants and $\left\{g_{j}\right\}$ a set of entire functions satisfying

$$
\sum_{j=1}^{p} \alpha_{j} g_{j}=1
$$

Then

$$
\sum_{j=1}^{p} \delta\left(0, g_{j}\right) \leqq p-1,
$$

where $\delta\left(0, g_{j}\right)$ denotes the Nevanlinna-deficiency.

LEMMA $2[6]$. Let $a_{0}(z), a_{1}(z), \cdots, a_{n}(z)$ be meromorphic functions and $g_{1}(z), \cdots, g_{n}(z)$ nonconstant entire functions. Further suppose that

$$
T\left(r, a_{j}\right)=o\left\{\sum_{\nu=1}^{n} m\left(r, e^{g_{\nu}(z)}\right)\right\}, \quad j=0,1, \cdots, n
$$

holds outside a set of finite linear measure. If an identity

$$
\sum_{\nu=1}^{n} a_{\nu}(z) \exp \left\{g_{\nu}(z)\right\}=a_{0}(z)
$$

holds, then we have an identity

$$
\sum_{\nu=1}^{n} c_{\nu} a_{\nu}(z) \exp \left\{g_{\nu}(z)\right\}=0
$$

where the constants $c_{\nu}, \nu=1, \cdots, n$, are not all zero.

This result has a more general form;

LEMMA 3. Let $a_{0}(z), a_{1}(z), \cdots, a_{n}(z)\left(a_{\nu} \neq 0, \nu \geqq 1\right)$ be meromorphic functions and $g_{1}(z), \cdots, g_{n}(z)$ nonconstant entire functions satisfying

$$
\underset{\substack{r \rightarrow \tilde{n}) \\(r \in \mathfrak{n})}}{\lim \sup _{1 \leqq \nu \leqq n}} \frac{\sum_{\nu=0}^{n} \log T\left(r, a_{\nu}\right)}{\operatorname{Max}_{1 \leqq} \log m\left(r, e^{g_{\nu}}\right)}<1,
$$

where $\Omega$ is a set of infinite linear measure. Suppose the identity

$$
\sum_{\nu=1}^{n} a_{\nu}(z) \exp \left\{g_{\nu}(z)\right\}=a_{0}(z)
$$

holds, then there exist $n$ constants $\left(c_{1}, \cdots, c_{n}\right) \neq(0, \cdots, 0)$ such that

$$
\sum_{\nu=1}^{n} c_{\nu} a_{\nu}(z) \exp \left\{g_{\nu}(z)\right\}=0 .
$$

This lemma is shown by quite a similar method to Lemma 2. Moreover a proof of Theorem 3 needs an estimation of Weierstrass products. 
LEMMA 4. Let $\left\{a_{\nu \mu}\right\}$ be $n$ sequences $(1 \leqq \mu \leqq n)$ of complex numbers satisfying $1 \leqq\left|a_{1 \mu}\right| \leqq\left|a_{2 \mu}\right| \leqq \cdots, \lim _{\nu \rightarrow \infty}\left|a_{\nu \mu}\right|=+\infty$ for each $\mu$. Then we can construct the Weierstrass products $P_{\mu}$ of the $\left\{a_{\nu \mu}\right\}(1 \leqq \mu \leqq n)$ with the following property:

There exists a set $\Omega$ in $[1, \infty)$ of infinite linear measure such that

$$
\frac{\sum_{\mu=1}^{n} \log m\left(r, P_{\mu}\right)}{\sum_{\mu=1}^{n} N\left(r, 1 / P_{\mu}\right)} \longrightarrow 0, \quad \text { as } r \rightarrow \infty, r \in \Omega \text {. }
$$

Mues [7] has proven this result for $n=1$, and his way to verification is still valid for this lemma by considering $\operatorname{Max}\left\{N_{\mu}(r)\right\}$ instead of $N(r)$ there, provided that $N_{\mu}(r)$ denotes a counting function for the $a_{\nu \mu}, \mu=1, \cdots, n$, and the maximum is taken over $\mu(1 \leqq \mu \leqq n)$.

\section{Proof of Theorem 1 .}

Our proof bears essentially on Ozawa's in [11] which depends on the impossibility of Borel's identity expressed as Lemma 1.

At first we note that the emptyness of $\left\{p_{n}\right\}$, the sequence of poles of $N$, reduces our proof to that for the case in which $N$ is an entire function. This has been settled by Ozawa, and there we find an exceptional form $e^{L}+A$, as in our theorem. Therefore we now assume $\left\{p_{n}\right\}$ to be not empty.

According to the assumptions, there are two meromorphic functions $f$ and $g$, together with entire functions $\alpha, \beta, \gamma$ and $\delta$ such that

$$
\begin{array}{ll}
f(z)=N(z) e^{\alpha(z)}, & f(z)-1=\{N(z)-1\} e^{\beta(z)}, \\
g(z)=N(z) e^{\gamma(z)}, & g(z)-d=\{N(z)-d\} e^{\delta(z)} .
\end{array}
$$

We may further assume that functions $f, g$ and $N$ are represented by the ratios $f_{1} / f_{2}, g_{1} / g_{2}$ and $N_{1} / N_{2}$ of entire functions with no common zero respectively. There is, however, no loss in generality in supposing that $f_{2}=g_{2}=N_{2}$ with the suitable choice of $\alpha$ and $\gamma$. Here in this case, $N_{2}$ is not of the form $e^{L}$ for entire $L$.

If $\left\{a_{n}\right\}$, the sequence of zeros of $N$, is empty, we can reduce this to the case for $\left\{p_{n}\right\}$ replacing $f, g, N$ and $d$ by $1 / f, 1 / g, 1 / N$ and $1 / d$ respectively. Thus $\left\{a_{n}\right\}$ is also supposed to be a nonempty set, noting that the second exception is $\left(e^{L}+A\right)^{-1}$.

A void $\left\{b_{n}\right\}$ of one-points of $N$ shows $N_{1}(z)-N_{2}(z)=e^{L(z)}$ with $L$ entire. From the relations

$$
\begin{array}{ll}
f_{1}(z)=N_{1}(z) e^{\alpha(z)}, & f_{1}(z)-N_{2}(z)=\left\{N_{1}(z)-N_{2}(z)\right\} e^{\beta(z)}, \\
g_{1}(z)=N_{1}(z) e^{\gamma(z)}, & g_{1}(z)-d N_{2}(z)=\left\{N_{1}(z)-d N_{2}(z)\right\} e^{\delta(z)} .
\end{array}
$$

we obtain 


$$
\begin{aligned}
& N_{2}(z)\left\{1-e^{\alpha(z)}\right\}=e^{L(z)}\left\{e^{\alpha(z)}-e^{\beta(z)}\right\}, \\
& N_{2}(z)\left\{d+(1-d) e^{\delta(z)}-e^{\gamma(z)}\right\}=e^{L(z)}\left\{e^{\gamma(z)}-e^{\delta(z)}\right\} .
\end{aligned}
$$

Hence we may assume that each of $e^{\alpha}, e^{\beta}, e^{\gamma}, e^{\delta}, e^{\alpha-\beta}$ and $e^{\gamma-\delta}$ is not identically equal to one, otherwise we have immediately the desired unicity of the given zero-one-pole or zero- $d$-pole set, $f=N$ or $g=N$. An elementary analysis shows $d+(1-d) e^{\delta}-e^{r} \neq 0$ and

$$
\frac{d\left(1-e^{\delta}\right)}{e^{\gamma}-e^{\delta}}=N=\frac{1-e^{\beta}}{e^{\alpha}-e^{\beta}},
$$

which implies that $\beta, \delta, \beta-\alpha$ and $\delta-\gamma$ are all nonconstant in the present case, and the identity

$$
d e^{\alpha-\beta}-d e^{\alpha-\beta+\delta}-e^{-\beta+\gamma}+e^{-\beta+\delta}+e^{\gamma}-(1-d) e^{\delta}=d .
$$

If all of these functions are nonconstant, then Lemma 1 gives an inequality

$$
\delta\left(0, e^{\alpha-\beta}\right)+\delta\left(0, e^{\alpha-\beta+\delta}\right)+\delta\left(0, e^{-\beta+\gamma}\right)+\delta\left(0, e^{-\beta+\delta}\right)+\delta\left(0, e^{\gamma}\right)+\delta\left(0, e^{\delta}\right) \leqq 5,
$$

by $d \neq 0,1$. This is, however, impossible since $\delta\left(0, e^{h}\right)=1$ for any nonconstant entire function $h$, and thus the left-hand side of this inequality must be equal to 6. Therefore at least one of these functions is a constant. Now we know that $\alpha-\beta$ and $\delta$ are not, so we shall discuss following four cases; (I ) $\gamma$, (II) $-\beta+\gamma$, (III) $-\beta+\delta$, or (IV) $\alpha-\beta+\delta$ is a constant $c$.

For Case (I), $e^{c \neq 1}$ and

$$
d e^{\alpha-\beta}-d e^{\alpha-\beta+\delta}-(1-d) e^{\delta}-e^{c} e^{-\beta}+e^{-\beta+\delta}=d-e^{c} .
$$

Firstly suppose that $\alpha-\beta+\delta$ is a constant $x$, thus it follows

$$
d e^{\alpha-\beta}-(1-d) e^{x} e^{-\alpha+\beta}-e^{c} e^{-\beta}+e^{x} e^{-\alpha}=d-e^{c}+d e^{x} .
$$

If $\alpha$ is not a constant, $d-e^{c}+d e^{x}$ must be equal to zero by Lemma 1 , since all functions here are nonconstant, therefore we have

$$
d e^{2 \alpha-\beta}-(1-d) e^{x} e^{\beta}-d\left(1+e^{x}\right) e^{\alpha-\beta}=-e^{x} .
$$

Lemma 1 also shows that $2 \alpha-\beta$ is a constant $y$ and this identity is

$$
(1-d) e^{x-y} e^{2 \alpha}+d\left(1+e^{x}\right) e^{y} e^{-\alpha}=d e^{y}+e^{x},
$$

which is a contradiction. Hence we see that $\alpha=y$ is a constant with $e^{y} \neq 1$, and thus identity (3) is

$$
\left(d e^{y}-e^{c}\right) e^{-\beta}-(1-d) e^{x-y} e^{\beta}=d-e^{c}+d e^{x}-e^{x-y},
$$

which gives a contradiction again, and the present assumption is denied in (2). We suppose secondly that $-\beta+\delta=x$ is a constant in (2) and obtain 


$$
d e^{\alpha-\beta}-d e^{x} e^{\alpha}-(1-d) e^{x} e^{\beta}-e^{c} e^{-\beta}=d-e^{c}-e^{x} .
$$

Here neither $\alpha-\beta+\delta$ nor $\alpha=\alpha-\beta+\delta-x$ is a constant, thus the lemma implies $e^{x}=d-e^{c}$ and

$$
d e^{\alpha}-d\left(d-e^{c}\right) e^{\alpha+\beta}-(1-d)\left(d-e^{c}\right) e^{2 \beta}=e^{c} .
$$

Then we see $\alpha+\beta$ is a constant $y$ and this identity is

$$
d e^{\alpha}-(1-d)\left(d-e^{c}\right) e^{2 y} e^{-2 \alpha}=e^{c}+d\left(d-e^{c}\right) e^{y},
$$

which is impossible, since now $\alpha$ is not a constant. Hence Lemma 1 gives $d=e^{c}$ in (2) and

$$
d e^{\alpha}-d e^{\alpha+\delta}-(1-d) e^{\beta+\delta}+e^{\delta}=d,
$$

which shows that at least one of $\alpha, \alpha+\delta$ and $\beta+\delta$ must be a constant. If $\alpha=x$ is so with $e^{x} \neq 1$, we have

$$
\left(1-d e^{x}\right) e^{\delta}-(1-d) e^{\beta+\delta}=d\left(1-e^{x}\right) .
$$

Since $\delta$ is not a constant, $\beta+\delta=y$ must be a constant and thus

$$
\left(1-d e^{x}\right) e^{\delta}=d\left(1-e^{x}\right)+(1-d) e^{y} \text {, or } e^{x}=1 / d \text { and } e^{y}=1 .
$$

From the above we see that $e^{\alpha}=1 / d, e^{\delta}=e^{-\beta}, e^{\gamma}=d$, and thus

$$
N=\frac{1-e^{\beta}}{e^{\alpha}-e^{\beta}}=\frac{d e^{\beta}-d}{d e^{\beta}-1}
$$

and of course $f=N / d$ and $g=d N$. This is the exceptional case asserted in our theorem. Next we assume that $\alpha$ is nonconstant but $\alpha+\delta=x$ is not. Then we can see that $e^{x}=-1$ and $d e^{2 \alpha}+(1-d) e^{\beta}=1$. While if $\beta+\delta$ is a constant $x$ and $\alpha$ is not, $(d-1) e^{\alpha+\beta}-d e^{\alpha}=-1$ is given similarly. In each case, Lemma 1 gives only a contradiction. These observations permit us to suppose that $\gamma$ is nonconstant.

In this manner, we can make sure of the fact that remained three cases produce nothing but a contradiction. Here we note that $e^{\alpha}=e^{2 \beta}, e^{\gamma}=d^{2} e^{\alpha}, e^{\delta}=$ $d e^{\beta}$ and $N=-e^{-\beta}$ may be derived for (III), although this is omitted now. Therefore we may assume that $\left\{b_{n}\right\}$ is a nonvoid set.

For the case in which the sequence of $d$-points of $N,\left\{c_{n}\right\}$, is empty we can discuss in much the same way and obtain the last exceptional form $\left(e^{L}-1\right)$ / $\left(e^{L}-d\right)$ with $L$ entire.

Now we shall observe the case where sequences $\left\{a_{n}\right\},\left\{b_{n}\right\},\left\{c_{n}\right\}$ and $\left\{p_{n}\right\}$ are all nonvoid. If $\alpha$ is a constant, then $f_{1}=C N_{1}$ with a constant $C=e^{\alpha}$. From the assumption, it follows $f_{1}\left(b_{n}\right)=N_{1}\left(b_{n}\right)=N_{2}\left(b_{n}\right) \neq 0$, therefore we have now $C=1$ and thus $f=N$. This is just the desired unicity of the given zero-onepole set, so that we may assume that $\alpha$ is nonconstant from now on. Also if $\beta$ is a constant, we have $f_{1}-N_{2}=C\left(N_{1}-N_{2}\right)$ with $e^{\beta}=C$ and obtain $C=1, f=N$ 
in consideration of $f_{1}\left(a_{n}\right)=N_{1}\left(a_{n}\right)=0$ and $N_{2}\left(a_{n}\right) \neq 0$. After this manner, all of $\alpha, \beta, \gamma$ and $\delta$ may be assumed to be nonconstant entire functions. We may further assume that so are both $\alpha-\beta$ and $\gamma-\delta$. In fact firstly each of $e^{\alpha-\beta} \equiv 1$ and $e^{\gamma-\delta} \equiv 1$ implies the desired result $f=N$ or $g=N$. In the second place either $e^{\alpha-\beta} \equiv a(\neq 1)$ or $e^{\gamma-\delta} \equiv b(\neq 1)$ for constants $a$ and $b$ gives

$$
N=\frac{1-e^{\beta}}{e^{\beta}(a-1)} \text { or } \frac{d\left(1-e^{\delta}\right)}{e^{\delta}(b-1)},
$$

respectively by our relations. Each case, however, contradicts the assumption that $N$ is meromorphic not entire. With these knowledges, we shall recur to the identity (1) and examine again all the possible cases only to get to a contradiction.

Thus we complete the proof of Theorem 1 .

\section{Proof of Theorem 2 .}

From the assumptions of this theorem we obtain the following equations;

$$
\begin{aligned}
& f(z)=g(z) e^{\alpha(z)}, \\
& f(z)-1=\{g(z)-1\} e^{\beta(z)},
\end{aligned}
$$

and

$$
f^{\prime}(z)-a=\left\{g^{\prime}(z)-a\right\} e^{r(z)},
$$

where $\alpha, \beta$ and $\gamma$ are entire functions. If one of $e^{\alpha}, e^{\beta}$ and $e^{\alpha-\beta}$ is identically equal to 1 , then (1) or (2) implies that $f$ and $g$ are identical. Therefore we may now assume that all of them fail and thus by (1) and (2)

$$
f(z)=\frac{e^{\alpha(z)}\left\{1-e^{\beta(z)}\right\}}{e^{\alpha(z)}-e^{\beta(z)}} \text { and } g(z)=\frac{1-e^{\beta(z)}}{e^{\alpha(z)}-e^{\beta(z)}} .
$$

Differentiating each both sides and substituting them into (3), we get the identity :

$$
\begin{aligned}
a e^{\alpha-\beta}+\beta^{\prime} e^{\alpha}-a e^{\alpha-\beta+\gamma} & +\beta^{\prime} e^{-\alpha+\gamma}+a e^{-\alpha+\beta}-\alpha^{\prime} e^{\beta}-a e^{-\alpha+\beta+\gamma} \\
& -\alpha^{\prime} e^{-\beta+\gamma}+\left(\alpha^{\prime}-\beta^{\prime}+2 a\right) e^{\gamma}=-\left(\alpha^{\prime}-\beta^{\prime}-2 a\right) .
\end{aligned}
$$

To prove the theorem, we shall make repeated use of Lemma 2 . In this case, Condition $\left(^{*}\right)$ is satisfied as follows ; for example, $\alpha^{\prime}$ is the logarithmic derivative of $e^{\alpha}$, and if $\alpha$ is nonconstant then we may use the theorem for a proximity function of the logarithmic derivative (see [5]), to get

$$
m\left(r, \alpha^{\prime}\right)=m\left\{r,\left(e^{\alpha}\right)^{\prime} / e^{\alpha}\right\}=o\left\{m\left(r, e^{\alpha}\right)\right\}
$$

outside a set of finite linear measure. Here $\alpha^{\prime}$ is entire so that this is true for the characteristic function, $T\left(r, \alpha^{\prime}\right)$. With respect to the identities appearing 
in our discussion, we need examining not only whether each exponential function is a constant or not, but also whether its coefficient $c_{j}$ is equal to zero or not. The detailed reason will be explained in the main part of our proof.

(A) We shall firstly proceed to the proof for the case in which $a=0$. Then identity (5) gives

$$
\beta^{\prime} e^{\alpha}+\beta^{\prime} e^{-\alpha+\gamma}-\alpha^{\prime} e^{\beta}+\left(\alpha^{\prime}-\beta^{\prime}\right) e^{\gamma}-\alpha^{\prime} e^{-\beta+\gamma}=\beta^{\prime}-\alpha^{\prime},
$$

which causes us to discuss following five cases; ( I ) $\alpha=x$, (II) $\beta=x$, (III) $\gamma=x$, (IV) $-\beta+\gamma=x,(\mathrm{~V})-\alpha+\gamma=x$ with a constant $x$.

Case $(I): \quad \alpha=x$ with $e^{x} \neq 1$.

Then we have $\beta^{\prime} e^{-x}\left(1-e^{x}\right) e^{\gamma}=\beta^{\prime}\left(1-e^{x}\right)$. Here we note that $\beta$ is not a constant, since otherwise (4) shows that both $f$ and $g$ are constants. Thus $e^{r}=e^{x}$ $=C$, say, by $e^{x} \neq 1$, so (1) and (4) give the desired relation

$$
f(z)=C g(z) \quad \text { with a constant } \quad C \neq 0,1,
$$

together with

$$
f(z)=\frac{C\left\{e^{\beta(z)}-1\right\}}{e^{\beta(z)}-C} \text { and } g(z)=\frac{e^{\beta(z)}-1}{e^{\beta(z)}-C} \text {. }
$$

Case (II): $\beta=x$ with $e^{x} \neq 1$.

This is the similar case to the above and gives

$$
f(z)-1=C\{g(z)-1\} \quad \text { with a constant } C \neq 0,1,
$$

and here

$$
f(z)=\frac{(1-C) e^{\alpha(z)}}{e^{\alpha(z)}-C} \text { and } g(z)=\frac{1-C}{e^{\alpha(z)}-C} .
$$

We may now assume that $\alpha$ and $\beta$ are both nonconstant from now on.

Case (III): $\gamma=x$.

In this case, (6) gives

$$
\beta^{\prime} e^{\alpha}-\alpha^{\prime} e^{\beta}+\beta^{\prime} e^{x} e^{-\alpha}-\alpha^{\prime} e^{x} e^{-\beta}=\left(\beta^{\prime}-\alpha^{\prime}\right)\left(e^{x}+1\right) .
$$

This identity obviously satisfies Condition $(*)$, so we have

$$
c_{1} \beta^{\prime} e^{\alpha+\beta}+c_{2} \alpha^{\prime} e^{2 \beta}+c_{3} \beta^{\prime} e^{-\alpha+\beta}=-c_{4} \alpha^{\prime}
$$

for the suitable constants $c_{j}$ as in Lemma 2. If $\alpha+\beta=y$ is a constant, then by (7) we have

$$
\left(e^{x}+e^{y}\right)\left(e^{-y} e^{\alpha}+e^{-\alpha}\right)=2\left(e^{x}+1\right)
$$


and thus $e^{y}=-e^{x}=1$ applying Lemma 1 , since $\alpha$ is not a constant. Here we obtain $e^{\alpha}=e^{-\beta}, e^{\gamma}=-1$ and

$$
\begin{aligned}
& f(z)+g(z)=1 \quad \text { with } \\
& f(z)=\frac{1}{1+e^{\beta(z)}} \quad \text { and } \quad g(z)=\frac{1}{1+e^{-\beta(z)}} .
\end{aligned}
$$

Then we may say that $\alpha+\beta$ is not a constant. While if $-\alpha+\beta=y$ is a constant with $e^{y} \neq 1,(7)$ gives $\alpha^{\prime}\left(1-e^{y}\right) e^{-\alpha}\left(e^{2 \alpha}-e^{x-y}\right)=0$, which is however impossible. Thus $-\alpha+\beta$ is not a constant, either. Now we may apply Lemma 2 to (8). In fact $\alpha+\beta, 2 \beta$ and $-\alpha+\beta$ are all nonconstant, and

$$
\begin{array}{ll}
T\left(r, c_{\imath} \beta^{\prime}\right)=o\left\{m\left(r, e^{\beta}\right)\right\}, & i=1,3, \\
T\left(r, c_{j} \alpha^{\prime}\right)=o\left\{m\left(r, e^{\alpha}\right)\right\}, & j=2,4,
\end{array}
$$

outside a set of finite linear measure. If at least two of the $c_{k}(k=1,2,3)$ are different from 0 , Condition $\left({ }^{*}\right)$ follows from Nevanlinna's fundamental estimates. For instance, $c_{1} \cdot c_{2} \neq 0$ gives

$$
\begin{aligned}
m\left(r, e^{\alpha}\right) & =\frac{1}{2} m\left(r, e^{2(\alpha+\beta)} / e^{2 \beta}\right) \leqq \frac{1}{2}\left\{m\left(r, e^{2(\alpha+\beta)}\right)+m\left(r, 1 / e^{2 \beta}\right)\right\} \\
& =\frac{1}{2}\left\{m\left(r, e^{2(\alpha+\beta)}\right)+m\left(r, e^{2 \beta}\right)+O(1)\right\} \\
& \leqq m\left(r, e^{\alpha+\beta}\right)+m\left(r, e^{2 \beta}\right)+O(1),
\end{aligned}
$$

as $r \rightarrow \infty$, excepting possibly a set of finite linear measure. Thus Lemma $2 \mathrm{im}$ plies an identity

$$
d_{1} \beta^{\prime} e^{\alpha+\beta}+d_{2} \alpha^{\prime} e^{2 \beta}+d_{3} \beta^{\prime} e^{-\alpha+\beta}=0
$$

for the suitable constants $d_{j}(j=1,2,3)$. This leads us to the conclusion

$$
\alpha^{\prime} e^{\alpha+\beta}=d \beta^{\prime}, \quad \beta^{\prime} e^{2 \alpha}=d \beta^{\prime}, \quad \text { or } \quad \beta^{\prime} e^{\alpha-\beta}=d \alpha^{\prime}
$$

with a nonzero constant $d$, although each gives a contradiction, which is quite the same when only one of the $c_{k}(k=1,2,3)$ is never zero. Hence we may say that $\gamma$ is nonconstant entire.

Case (IV): $-\beta+\gamma=x$.

Then (6) is of the form;

$$
\beta^{\prime} e^{\alpha}+\left\{\left(e^{x}-1\right) \alpha^{\prime}-e^{x} \beta^{\prime}\right\} e^{\beta}+\beta^{\prime} e^{x} e^{-\alpha+\beta}=\left(e^{x}-1\right) \alpha^{\prime}+\beta^{\prime} .
$$

If $-\alpha+\beta=y$ is a constant with $e^{y} \neq 1$, then it follows from this identity $\alpha^{\prime}\left(1-e^{y}\right) e^{\alpha}=\alpha^{\prime} e^{x}\left(1-e^{y}\right)$ which is evidently a contradiction. Hence $-\alpha+\beta$ is not a constant in the present case. Then we see that Condition (*) holds for (9) 
and so we have

$$
c_{1} \beta^{\prime} e^{\alpha-\beta}+c_{3} \beta^{\prime} e^{-\alpha}=-c_{2}\left\{\left(e^{x}-1\right) \alpha^{\prime}-e^{x} \beta^{\prime}\right\}
$$

with constants $\left(c_{1}, c_{2}, c_{3}\right) \neq(0,0,0)$. Here if $\left(e^{x}-1\right) \alpha^{\prime}-e^{x} \beta^{\prime} \equiv 0$ in (9), $e^{\alpha}+e^{x} e^{-\alpha+\beta}$ $=e^{x}+1$ by $\beta^{\prime} \neq 0$. Lemma 1 implies $e^{x}=-1$ and $e^{\beta}=e^{2 \alpha}$, so that $e^{\gamma}=-e^{\beta}=-e^{2 \alpha}$ and thus

$$
\{f(z)-1\}\{g(z)-1\}=1
$$

with functions

$$
f(z)=1+e^{\alpha(z)} \text { and } g(z)=1+e^{-\alpha(z)} .
$$

Next we suppose $\left(e^{x}-1\right) \alpha^{\prime}-e^{x} \beta^{\prime} \not \equiv 0$. If one of $c_{1}$ and $c_{3}$ is equal to zero, we have

$$
d \beta^{\prime} e^{-\alpha}=\left\{\left(e^{x}-1\right) \alpha^{\prime}-e^{x} \beta^{\prime}\right\} \quad \text { or } \quad d \beta^{\prime} e^{\alpha-\beta}=\left\{\left(e^{x}-1\right) \alpha^{\prime}-e^{x} \beta^{\prime}\right\}
$$

with a nonzero constant $d$, which implies $e^{x}=-1$ and either

$$
e^{2 \alpha}+(d-1) e^{\beta}=d \text { or }(d+1) e^{\beta}-e^{-2 \alpha+2 \beta}=d,
$$

substituting into (9) and applying Lemma 1. Each identity contradicts this Lemma 1. Hence we see that $c_{1} \cdot c_{3} \neq 0$ and apply Lemma 2 to get $e^{\beta}=d e^{2 \alpha}$ with a nonzero constant $d$. Again substituting this into (9), Lemma 1 gives

$$
2\left(d e^{x}+1\right) e^{\alpha}-d\left(e^{x}+1\right) e^{2 \alpha}=e^{x}+1
$$

and thus $e^{x}+1=0$ and $d=1$, or $e^{\beta}=e^{2 \alpha}$ and $e^{\gamma}=-e^{2 \alpha}$, which is mentioned above. Hence we may assume that $-\beta+\gamma$ is also not a constant now.

Case $(V):-\alpha+\gamma=x$.

This is the case that we can discuss in much the same way as Case (IV), and see that only the possible one is the following; $e^{x}=-1$ and $e^{\beta}=e^{\alpha-\beta}$,

$$
f(z)=-e^{\beta(z)} \text { and } g(z)=-e^{-\beta(z)}
$$

and their relationship is

$$
f(z) \cdot g(z)=1 \text {. }
$$

Hence $-\alpha+\gamma$ is supposed to be not a constant, either.

Now we return to the initial identity (6) and apply Lemma 2 there to get

$$
c_{2} \alpha^{\prime} e^{-\alpha+\beta}+c_{3}\left(\alpha^{\prime}-\beta^{\prime}\right) e^{-\alpha+\gamma}+c_{4} \alpha^{\prime} e^{-\alpha-\beta+\gamma}+c_{5} \beta^{\prime} e^{-2 \alpha+\gamma}=-c_{1} \beta^{\prime},
$$

for suitable constants $c_{1}, \cdots, c_{5}$ not all zero. We now need to discuss again the following cases: (VI) $-\alpha+\beta=x$, (VII) $-\alpha-\beta+\gamma=x$, and (VIII) $-2 \alpha+\gamma=x$ with a constant $x$. 
Case $(V I):-\alpha+\beta=x$ with $e^{x} \neq 1$.

Then (6) has a form, $\beta^{\prime}\left(e^{x}-1\right) e^{\beta}=e^{x} \beta^{\prime}\left(e^{x}-1\right) e^{-\beta+\gamma}$ so that we obtain $e^{\alpha}=C e^{\beta}$ and $e^{\gamma}=C e^{2 \beta}$ with $C=e^{-x}$. Therefore we derive the final relationship

$$
\{(C-1) f(z)-C\}\{(C-1) g(z)+1\}=-C,
$$

which is satisfied by

$$
f(z)=\frac{C\left(1-e^{\beta(z)}\right)}{C-1} \text { and } g(z)=\frac{e^{-\beta(2)}-1}{C-1},
$$

and we may assume that $\alpha-\beta$ is also not a constant, and thus $\alpha^{\prime}-\beta^{\prime} \neq \equiv$.

Case $(V I I): \alpha+\beta-\gamma=x$.

Identity (6) gives now

$$
\left(\beta^{\prime}-\alpha^{\prime} e^{-x}\right) e^{\alpha}+\left(\alpha^{\prime}-\beta^{\prime}\right) e^{-x} e^{\alpha+\beta}+e^{-x}\left(\beta^{\prime}-\alpha^{\prime} e^{x}\right) e^{\beta}=\beta^{\prime}-\alpha^{\prime} .
$$

Here we note that $\alpha+\beta=\gamma+x$ is not a constant, and both $\beta^{\prime}-\alpha^{\prime} e^{-x}$ and $\beta^{\prime}-$ $\alpha^{\prime} e^{x}$ do not vanish identically since otherwise $e^{x}= \pm 1$ and $\alpha^{\prime} \pm \beta^{\prime} \equiv 0$ or $\alpha \pm \beta$ is a constant. There is no loss in generality supposing $\beta^{\prime}-\alpha^{\prime} e^{-x}$ not to vanish identically. Applying the lemma to (11), there exist constants $c_{1}, c_{2}$ and $c_{3}$ not all zero satisfying

$$
c_{2}\left(\alpha^{\prime}-\beta^{\prime}\right) e^{\beta}+c_{3}\left(e^{x} \alpha^{\prime}-\beta^{\prime}\right) e^{-\alpha+\beta}=-c_{1}\left(\alpha^{\prime}-e^{x} \beta^{\prime}\right) .
$$

If $e^{x} \alpha^{\prime}-\beta^{\prime} \equiv 0$ in $(11)$,

$$
e^{-x}\left(e^{2 x}-1\right) \alpha^{\prime} e^{\alpha}+e^{-x}\left(1-e^{x}\right) \alpha^{\prime} e^{\alpha+\beta}=\left(e^{x}-1\right) \alpha^{\prime},
$$

so that $e^{x}=1$, which gives a contradiction, $\alpha^{\prime}-\beta^{\prime} \equiv 0$. At last we see that both $e^{x} \alpha^{\prime}-\beta^{\prime} \neq 0$ and $\alpha^{\prime}-\beta^{\prime} e^{x} \neq 0$. The consequences of (12) are therefore,

and

$$
\left(e^{x} \alpha^{\prime}-\beta^{\prime}\right) e^{-\alpha+\beta}=d\left(\alpha^{\prime}-e^{x} \beta^{\prime}\right), \quad\left(\alpha^{\prime}-\beta^{\prime}\right) e^{\beta}=d\left(\alpha^{\prime}-e^{x} \beta^{\prime}\right)
$$

$$
\left(\alpha^{\prime}-\beta^{\prime}\right) e^{\alpha}=d\left(e^{x} \alpha^{\prime}-\beta^{\prime}\right), \quad \text { with a nonzero constant } d \text {. }
$$

In each case we can reduce (11) to an identity with constant coefficients and derive a contradiction by Lemma 1 . Hence we see also that $\alpha+\beta-\gamma$ must be nonconstant.

Case (VIII): $2 \alpha-\gamma=-x$

In this last examination, (6) is the identity of the form

$$
\beta^{\prime}\left(e^{x}+1\right) e^{\alpha}-\alpha^{\prime} e^{\beta}+\left(\alpha^{\prime}-\beta^{\prime}\right) e^{x} e^{2 \alpha}-\alpha^{\prime} e^{x} e^{2 \alpha-\beta}=\beta^{\prime}-\alpha^{\prime},
$$

and $2 \alpha-\beta=-x-\beta+\gamma$ is not a constant here, so that (13) satisfies Condition (*). 
The coefficient $e^{x}+1=0$ gives

$$
-\alpha^{\prime} e^{\beta}-\left(\alpha^{\prime}-\beta^{\prime}\right) e^{2 \alpha}+\alpha^{\prime} e^{2 \alpha-\beta}=\beta^{\prime}-\alpha^{\prime},
$$

from which Lemma 2 implies an identity, $c_{2}\left(\alpha^{\prime}-\beta^{\prime}\right) e^{2 \alpha-\beta}+c_{3} \alpha^{\prime} e^{2 \alpha-2 \beta}=-c_{1} \alpha^{\prime}$, where the $c_{j}(j=1,2,3)$ are constant not all zero. The consequences of this identity due to Lemma 2 are

$$
e^{2(\alpha-\beta)}=d, \quad\left(\alpha^{\prime}-\beta^{\prime}\right) e^{\alpha-\beta}=d \alpha^{\prime} e^{-\alpha} \quad \text { and } \quad\left(\alpha^{\prime}-\beta^{\prime}\right) e^{\beta-\alpha}=d \alpha^{\prime} e^{-\alpha}
$$

with a nonzero constant $d$. The first is evidently a contradiction. Integrating the second equation, we have the result that $e^{\alpha-\beta}+d e^{-\alpha}$ is a constant, which contradicts our assumption according to Lemma 1 . The same holds for the last equation. Thus we have $e^{x}+1 \neq 0$, however in this case, only a similar contradiction is derived in much the same way. Here we note that when there appears an identity inapplicable for Lemma 2, for example,

$$
\beta^{\prime}=A \alpha^{\prime} e^{-\alpha+\beta}+B \alpha^{\prime} e^{\alpha-\beta} \quad \text { with nonzero constants } A \text { and } B,
$$

we shall substitute this to (13) and reduce it to an identity with constant coefficients, which is a matter of Lemma 1. In like manner, we see that $2 \alpha-\gamma$ is nonconstant and so is $2 \beta-\gamma$, and come to an end of the examination.

Recurring to the identity (10) again and supposing constants $c_{j}(2 \leqq j \leqq 5)$ to be different from zero, there exist the constants $d_{k}(k=1, \cdots, 4)$ of the same property satisfying

$$
d_{1} \alpha^{\prime} e^{\alpha+\beta-\gamma}+d_{2}\left(\alpha^{\prime}-\beta^{\prime}\right) e^{\alpha}+d_{3} \alpha^{\prime} e^{\alpha-\beta}=-d_{4} \beta^{\prime} .
$$

If either $d_{2}$ or $d_{3}$ is equal to zero, we reduce (10) to the identity with constant coefficients by substitution to get a contradiction. Otherwise it follows further

$$
u_{1} \alpha^{\prime} e^{\alpha+\beta-\gamma}+u_{2}\left(\alpha^{\prime}-\beta^{\prime}\right) e^{\alpha}+u_{3} \alpha^{\prime} e^{\alpha-\beta}=0,
$$

where $u_{1}, u_{2}$ and $u_{3}$ are not all zero. This gives us the following consequence: for a nonzero constant $d$,

$$
\begin{aligned}
& e^{2 \beta-\gamma}=d, \text { or }\left(\alpha^{\prime}-\beta^{\prime}\right) e^{\beta}=d \alpha^{\prime}, \quad \text { or }\left(\alpha^{\prime}-\beta^{\prime}\right) e^{-\beta+\gamma}=d \alpha^{\prime}, \\
& \text { or else } \beta^{\prime}=\alpha^{\prime}\left(A e^{\beta-\gamma}+B e^{-\beta}+1\right) \text { with nonzero constants } A \text { and } B .
\end{aligned}
$$

The first two are both immediate contradictions. Remained two results reduce (6) to identities with constant coefficients, and this brings us to the conclusion with a contradiction again. For the other cases, the analogous reasonings hold and give nothing. In this way we find a completion of the proof for the case: $a=0$.

(B) Next we set about the proof for the case: $a \neq 0$. Recall the identity (5) and note that in the present case we can nevermore reduce this identity to the 
one with constant coefficients through a substitution of some equation about $\alpha^{\prime}$ and $\beta^{\prime}$. Instead of this we have hypothesized that both $f$ and $g$ are of hyperorder less than one. Then Condition $\left(^{*}\right)$ always holds, if every exponential function is not identically constant. In fact, suppose otherwise with respect to $\alpha^{\prime}-\beta^{\prime}+2 a$ for example, that is, there exist a nonconstant entire function $h$ say, as in (5) and a set $\Omega$ of infinite linear measure such that

$$
\limsup _{\substack{r \rightarrow \infty \\(r \in \Omega)}} \frac{T\left(r, \alpha^{\prime}-\beta^{\prime}+2 a\right)}{m\left(r, e^{h}\right)}>0 .
$$

This is, however, impossible since $T\left(r, \alpha^{\prime}-\beta^{\prime}+2 a\right) \leqq r^{\varepsilon}, 1>\varepsilon>0$ for sufficiently large $r$ and $m\left(r, e^{h}\right) \geqq m\left(r, e^{c z}\right)=|c| r / \pi$ for some nonzero constant $c$ (see [5], page 7). Therefore we can proceed to the discussion in much the same way as in the previous Case (A). Here only the possible cases are introduced without repeating such the complicated examinations as in (A).

$$
\text { Case }(I): \quad \alpha-\beta=x \text { is a constant with } e^{x} \neq 1 \text {. }
$$

Here we may assume that both $\alpha$ and $\beta$ are nonconstant, otherwise functions $f$ and $g$ are reduced to constants. Our initial identity (5) gives now

$$
\beta^{\prime} e^{\beta}-a e^{-x}\left(e^{x}-1\right) e^{\gamma}-e^{-x} \beta^{\prime} e^{-\beta+\gamma}=-a e^{-x}\left(e^{x}-1\right) .
$$

If $\gamma$ is a constant $c$, say, $\beta^{\prime}\left(e^{\beta}-e^{c-x} e^{-\beta}\right)=a e^{-x}\left(e^{x}-1\right)\left(e^{c}-1\right)$, which shows that $\beta$ is a constant. Otherwise, we suppose that $-\beta+\gamma$ is a constant $c$. Then (6) also gives an identity $\left\{\beta^{\prime}-a e^{c-x}\left(e^{x}-1\right)\right\} e^{\beta}=e^{c-x}\left\{\beta^{\prime}-a e^{-c}\left(e^{x}-1\right)\right\}$. The entire function $\beta$ is not a constant, and

$$
m\left(r, \beta^{\prime}+\text { constant }\right)=o\left\{m\left(r, e^{\beta}\right)\right\}
$$

outside a set of finite linear measure, therefore we must deduce

$$
\beta^{\prime}-a e^{c-x}\left(e^{x}-1\right) \equiv 0 \quad \text { and } \quad \beta^{\prime}-a e^{-c}\left(e^{x}-1\right) \equiv 0 \quad \text { simultaneously, }
$$

So that $e^{c}= \pm e^{(1 / 2) x}$ and $\beta^{\prime}(z) \equiv \pm a e^{-(1 / 2) x}\left(e^{x}-1\right)$, double signs in same order. Then $e^{\alpha}=e^{x} e^{\beta}, e^{\gamma}= \pm e^{(1 / 2) x} e^{\beta}$ and als (4) give

$$
f(z)=\frac{e^{x}\left\{1-e^{\beta(z)}\right\}}{e^{x}-1} \text { and } g(z)=\frac{e^{-\beta(z)}-1}{e^{x}-1},
$$

which are both entire of order exactly one. Their relationship is

$$
\{(C-1) f(z)-C\}\{(C-1) g(z)+1\}=-C
$$

with a constant $C=e^{x} \neq 0,1$. Next suppose that $-\beta+\gamma$ is not a constant in (6) and apply Lemma 2 there to get $c_{1} \beta^{\prime} e^{2 \beta-\gamma}+c_{2} e^{\beta}=-c_{3} \beta^{\prime}$ with the constants $c_{j}$ $(j=1,2,3)$ not all zero. Then we see that $2 \beta-\gamma$ must be a constant from (6) and arrive at a contradiction, so that $\alpha-\beta$ may be supposed not to be a constant from now on. 
Case (II): $\alpha-\beta+\gamma=x$ is a constant.

Then we obtain the starting identity

$$
\begin{aligned}
a e^{\alpha-\beta} & +\beta^{\prime} e^{\alpha}+\beta^{\prime} e^{x} e^{-2 \alpha+\beta}+\left\{\alpha^{\prime}-\beta^{\prime}+\alpha\left(2+e^{-x}\right)\right\} e^{x} e^{-\alpha-3} \\
& -\alpha^{\prime} e^{\beta}-a e^{x} e^{-2 \alpha+2 \beta}-\alpha^{\prime} e^{x} e^{-\alpha}=-\left\{\alpha^{\prime}-\beta^{\prime}-a\left(2+e^{x}\right)\right\} .
\end{aligned}
$$

If $-2 \alpha+\beta=c$ is a constant, then Identity (7) is

$$
\begin{aligned}
-\left(\alpha^{\prime}-a e^{-x-c}\right) e^{x} e^{-\alpha}- & \left\{\left(1-2 e^{-x-c}\right) \alpha^{\prime}-a\left(2+e^{-x}\right)\right\} e^{x+c} e^{\alpha} \\
- & \left(\alpha^{\prime}+a e^{x+c}\right) e^{c} e^{2 \alpha}=\left(1-2 e^{x+c}\right) \alpha^{\prime}+a\left(2+e^{x}\right),
\end{aligned}
$$

from which we must derive that all coefficients of exponential functions here vanish identically. In fact otherwise its consequence by the lemma is such a contradiction ;

$$
e^{n \alpha}=\frac{\left(A_{1} \alpha^{\prime}+B_{1}\right)}{\left(A_{2} \alpha^{\prime}+B_{2}\right)} \quad \text { with an integer } n(\neq 0) \text { and constants } A_{\jmath}, B_{j}(j=1,2) .
$$

Thus we obtain $e^{c}=1, e^{2 x}=-1$, so that $e^{\beta}=e^{2 \alpha}$ and $e^{\gamma}= \pm i e^{\alpha}$, by which (4) gives

$$
f(z)=1+e^{\alpha(z)} \quad \text { and } \quad g(z)=1+e^{-\alpha(z)}
$$

with $\alpha^{\prime}(z) \equiv \mp i a$. They are both entire of order exactly one, and related by

$$
\{f(z)-1\} \cdot\{g(z)-1\}=1 \text {. }
$$

The similar is given for $-\alpha+\beta+\gamma=x$ and $\alpha-2 \beta=c$, that is,

$$
f(z)=-e^{\beta(z)} \text { and } g(z)=-e^{-\beta(z)}
$$

with $\beta^{\prime}(z) \equiv \pm i a$, and their relationship

$$
f(z) \cdot g(z)=1 .
$$

Then we may suppose that $-2 \alpha+\beta$ is not a constant in (7) and discuss in the usual way only to have a contradiction. We shall make it sure that the other linear combinations of $\alpha, \beta$ and $\gamma$ than the aboves cannot be constants in this manner, and then Lemma 2 and our restrictions on the orders of $\alpha, \beta$ and $\gamma$ lead us to a completion of the proof.

\section{Proof of Theorem 3.}

Our assumptions of this theorem imply immediately

$$
f(z)^{n}-1=\left\{g(z)^{n}-1\right\} e^{\alpha(z)} \text { and } f(z)=g(z) e^{\beta(z)}
$$

with entire functions $\alpha$ and $\beta$. We now distinguish three cases following; 
Case 1) $\beta$ is a constant with $e^{\beta} \equiv C \in C-\{0\}$.

Then we have $\left(e^{\alpha(z)}-C^{n}\right) g(z)^{n}=e^{\alpha(z)}-1$, so that either $e^{\alpha(z)} \equiv C^{n}=1$ or

$$
g(z)^{n}=\frac{e^{\alpha(z)}-1}{e^{\alpha(z)}-C^{n}} .
$$

Since $n \geqq 2$, this function cannot assume a simple zero and a simple pole. Therefore $C^{n}$ must be equal to 1 . In each case we have

$$
f(z)=C g(z), \quad C^{n}=1 .
$$

Case 2) $\alpha$ is a constant with $e^{\alpha} \equiv C \in C-\{0\}$, and $\beta$ is not a constant.

In this case we have also

$$
g(z)^{n}=\frac{1-C}{\left(e^{n \beta(z)}-C\right)},
$$

which gives $C=1$ and thus $f(z)^{n}=g(z)^{n}$ by the same reason as the above.

Case 3) Both $\alpha$ and $\beta$ are nonconstant.

Then we may set

$$
g(z)^{n}=\frac{e^{-\alpha(z)}-1}{e^{n \beta(z)-\alpha(z)}-1}=\left(\frac{N(z)}{P(z)}\right)^{n} e^{H(z)},
$$

with entire functions $N, P, H$, provided that $N$ and $P$ do not vanish identically and posess no common zero. Thus $N^{n}$ and $P^{n}$ assume the value 0 possibly at a multiple zero of $e^{-\alpha}-1$ and $e^{n \beta-\alpha}-1$ respectively.

In order to complete our proof we now apply Lemma 3 to the identity

$$
P^{n} e^{-\alpha}-N^{n} e^{n \beta-\alpha+H}+N^{n} e^{H}=P^{n} .
$$

To do this we need to make sure of the followings: in the first place,

$$
m\left(r, e^{-\alpha}\right) \sim m\left(r, e^{n \beta-\alpha}\right), \quad r \notin E
$$

where $E$ is a set of finite linear measure. (After this $E$ denotes such an exceptional set and may not always be the same. Here $S_{1}(r) \sim S_{2}(r)$ means that their ratio tends to 1 as $r \rightarrow \infty$.) And secondly

$$
\log ^{+} m\left(r, N^{n}\right)+\log ^{+} m\left(r, P^{n}\right)=o\left\{\log ^{+} m\left(r, e^{-\alpha}\right)\right\},
$$

in a set of infinite linear measure. (2) can be given by estimations

$$
\begin{aligned}
& N\left(r, \frac{1}{e^{-\alpha}-1}\right)+O\left\{\log ^{+} m\left(r, e^{-\alpha}\right)+\log r\right\} \\
= & N\left(r, \frac{1}{e^{n \beta-\alpha}-1}\right)+O\left\{\log ^{+} m\left(r, e^{n \beta-\alpha}\right)+\log r\right\}, \quad r \notin E
\end{aligned}
$$


and

$$
\begin{aligned}
& m\left(r, \frac{1}{e^{-\alpha}-1}\right)=O\left\{\log ^{+} m\left(r, e^{-\alpha}\right)+\log r\right\}, \quad r \notin E, \\
& m\left(r, \frac{1}{e^{n \beta-\alpha}-1}\right)=O\left\{\log ^{+} m\left(r, e^{n \alpha-\beta}\right)+\log r\right\}, \quad r \notin E .
\end{aligned}
$$

The first one is a consequence of the fact that all simple zeros of $e^{-\alpha}-1$ coincide with those of $e^{n \beta-\alpha}-1$. While Nevanlinna's first and second fundamental theorems give the last two estimations. We note that (2) shows $n \beta-\alpha$ is not a constant.

Now if $P^{n}$, for example, has at most a finite number of zeros, we may choose this as a polynomial, so that $m\left(r, P^{n}\right)=O(\log r)$, which is a quantity of no matter in (3). Thus we may suppose that both $P^{n}$ and $N^{n}$ posess infinitely many zeros and satisfy the hypotheses of Lemma 4 . Then there exists a set $\Omega$ of infinite linear measure such that for Weierstrass products $P^{n}$ and $N^{n}$,

$$
\frac{\log m\left(r, P^{n}\right)+\log m\left(r, N^{n}\right)}{N\left(r, 1 / P^{n}\right)+N\left(r, 1 / N^{n}\right)} \longrightarrow 0 \quad \text { as } \quad r \rightarrow \infty, r \in \Omega \text {. }
$$

Since $N\left(r, 1 / P^{n}\right)$ and $N\left(r, 1 / N^{n}\right)$ are bounded upwards by counting functions for multiple zeros of $e^{-\alpha}-1$ and $e^{n \beta-\alpha}-1$ respectively, we have

$$
\begin{aligned}
N\left(r, 1 / P^{n}\right)+N\left(r, 1 / N^{n}\right)= & O\left\{\log ^{+} m\left(r, e^{-\alpha}\right)\right. \\
& \left.+\log ^{+} m\left(r, e^{n \beta-\alpha}\right)+\log r\right\}, \quad r \notin E .
\end{aligned}
$$

These two results together with (2) imply (3).

Next we need to examine the case where either $n \beta-\alpha+H$ or $H$ is a constant, before we apply directly Lemma 3 to our identity (1). If $n \beta-\alpha+H \equiv c$ is a constant, (1) gives

$$
P^{n} e^{-\alpha}+N^{n} e^{c} e^{-n \beta+\alpha}=P^{n}+e^{c} N^{n} .
$$

We can apply the lemma here and get $N^{n} e^{c}=d P^{n} e^{n \beta-2 \alpha}$ for a nonzero constant $d$. Substituting this into (4) we obtain $e^{\alpha}+d e^{n \beta-\alpha}=1+d$, which gives $d=-1$ and $e^{n \beta-\alpha}=e^{\alpha}$ by Lemma 1 , so that

$$
g(z)^{n}=\frac{e^{-\alpha(z)}-1}{e^{\alpha(z)}-1}=-e^{-\alpha(z)} \text { and } f(z)^{n}=g(z)^{n} e^{n \beta(z)}=-e^{\alpha(z)} .
$$

While $H(z) \equiv c+\pi i, c$ a constant, makes again the identity (4) from (1).

Then we may assume that both $n \beta-\alpha+H$ and $H$ are nonconstant and apply Lemma 3 to (1) to get

$$
d_{1} P^{n} e^{-\alpha}+d_{2} N^{n} e^{H}+d_{3} N^{n} e^{n \beta-\alpha+H}=0
$$

with the constants $d_{j}(j=1,2,3),\left(d_{1}, d_{2}, d_{3}\right) \neq(0,0,0) . d_{1}=0$ implies that $d_{2} \cdot d_{3}$ $\neq 0$ and $e^{n \beta-\alpha}$ is a constant, which is absurd. $d_{2}=0$ gives $d_{1} \cdot d_{3} \neq 0$ and $N^{n} e^{n \beta+H}$ $=d P^{n}$ for $d \in C-\{0\}$. The initial identity (1) together with this leads us to 
the result $e^{\alpha}-d e^{-n \beta+\alpha}=1-d$, or $d=1$ and $e^{n \beta-\alpha}=e^{-\alpha}$ according to Lemma 1 . Then we have $f^{n}=g^{n} \equiv 1$. If $d_{3}=0, N^{n} e^{H}=-d P^{n} e^{-\alpha}$ for $d \in C-\{0\}$, which derives the same as in the case where $d_{2}=0$ from (1). Hence we may choose the $d_{j}$ by $d_{1} d_{2} d_{3} \neq 0$ and obtain the following from (5),

$$
P^{n} e^{-\alpha-H}+d N^{n} e^{n \beta-\alpha}=\tilde{d} N^{n}, \quad d, \tilde{d} \in \boldsymbol{C}-0 .
$$

If $\alpha+H$ is nonconstant here, it follows from the lemma $N^{n} e^{H}=-d N^{n} e^{-n \beta}$ which has been discussed above. Suppose $\alpha+H \equiv c$ is a constant. Then (1) gives the identity

$$
P^{n} e^{\alpha}+N^{n} e^{c} e^{n \beta-\alpha}=P^{n}+N^{n} e^{c},
$$

which however concludes similarly to (4). Now we have checked up on all the possibilities and complete the proof.

\section{Proof of Theorem 4 .}

From the assumptions of Theorem 4, we obtain the usual equations

$$
\begin{aligned}
& f(z)-1=\{g(z)-1\} e^{\alpha(z)}, \\
& f(z)+1=\{g(z)+1\} e^{\beta(z)} \text { and } \\
& \{f(z)-a\}\{f(z)-b\}=\{g(z)-a\}\{g(z)-b\} e^{\gamma(z)}
\end{aligned}
$$

for entire functions $\alpha, \beta$ and $\gamma$. Now we may assume that each of $e^{\alpha(z)}$, $e^{\beta(z)}$ and $e^{\alpha(z)-\beta(z)}$ is not identically equal to one, otherwise (1) and (2) show that $f$ and $g$ are unique. Then (1) and (2) also give

$$
f(z)=\frac{2 e^{\alpha(z)+\beta(z)}-e^{\alpha(z)}-e^{\beta(z)}}{e^{\alpha(z)}-e^{\beta(z)}}, \quad g(z)=\frac{e^{\alpha(z)}+e^{\beta(z)}-2}{e^{\alpha(z)}-e^{\beta(z)}},
$$

and together with (3)

$$
\begin{aligned}
4 e^{2 \beta} & -2(a+b+2) e^{\beta}-(1-a)(1-b) e^{\gamma}-2(a b-1) e^{-\alpha+\beta}+2(a+b-2) e^{-\alpha+2 \beta} \\
& +2(a b-1) e^{-\alpha+\beta+\gamma}-2(a+b-2) e^{-\alpha+\gamma}+(1-a)(1-b) e^{-2 \alpha+2 \beta} \\
& -(1+a)(1+b) e^{-2 \alpha+2 \beta+\gamma}+2(a+b+2) e^{-2 \alpha+\beta+\gamma}-4 e^{-2 \alpha+\gamma}=-(1+a)(1+b) .
\end{aligned}
$$

For want of space, we shall now introduce only the possible combinations with $\alpha, \beta, \gamma$, and the relations between $f$ and $g$ as well as their representations, excepting the examinations which are quite similar to the proof of our first theorem.

Case (I): $\beta=x$ is a constant with $e^{x} \neq 1$.

Then (5) gives an identity 


$$
\begin{aligned}
& 2 e^{x}\left\{(a+b-2) e^{x}-(a b-1)\right\} e^{-\alpha}+2\left\{(a b-1) e^{x}-(a+b-2)\right\} e^{-\alpha+\gamma} \\
& \quad+(1-a)(1-b) e^{2 x} e^{-2 \alpha}-\left\{(1+a)(1+b) e^{2 x}-2(a+b+2) e^{x}+4\right\} e^{-2 \alpha+\gamma} \\
& \quad-(1-a)(1-b) e^{\gamma}=\left(-2 e^{x}+1+a\right)\left(2 e^{x}-1-b\right) .
\end{aligned}
$$

Here we note that $\alpha$ is not a constant now, since otherwise both $f$ and $g$ are constants according to (4). It is immediately shown that both $-\alpha+\gamma$ and $-2 \alpha+\gamma$ are nonconstants by Lemma 1 , and the same is valid for $\gamma$ under the restriction that $a$ and $b$ are different from \pm 1 . Therefore Lemma 1 implies that the right-hand side is equal to 0 , that is, $e^{x}=1 / 2(a+1)$ or $1 / 2(b+1)$. We may choose the former, with no loss of generality, so that the consequence of (6) is

$$
\begin{aligned}
2(a+1)(a-b) e^{-\alpha-\gamma} & -(1-b)(1+a)^{2} a^{-2 \alpha-\gamma}+4(a b+2 b-3) e^{-\alpha} \\
& -(a+3)\{(a-3)+b(a+1)\} e^{-2 \alpha}=4(b-1),
\end{aligned}
$$

which shows that either $\alpha+\gamma$ or $2 \alpha+\gamma$ must be a constant $y$. At the first place, we suppose so is $\alpha+\gamma$, that is,

$$
\begin{gathered}
\left\{4(a b+2 b-3)+(b-1)(1+a)^{2} e^{-y}\right\} e^{-\alpha}-(a+3)\{(a-3)+b(a+1)\} e^{-2 \alpha} \\
=4(b-1)-2(a+1)(a-b) e^{-y},
\end{gathered}
$$

from which it follows that the coefficients are equal to $0 . a+3=0$ is evidently a contradiction. While if $(a-3)+b(a+1)=0$, or $(1+a)(1+b)=4$, then an elementary calculation gives $a^{2}+4 a+7=0$, so that $a=-2 \pm \sqrt{3} i$ and $b=-2 \mp \sqrt{3} i$, double signs in same order. Then $e^{\beta}=e^{x}=1 / 2(a+1)=1 / 2(-1 \pm \sqrt{3} i)=\omega$, say and $e^{y}=$ $-1 / 4(a+1)(a+3)=1$ or $e^{\gamma}=e^{-\alpha}$, so that (4) yields

$$
f(z)=\frac{(2 \omega-1) e^{\alpha(z)}-\omega}{e^{\alpha(z)}-\omega} \text { and } g(z)=\frac{e^{\alpha(z)}+\omega-2}{e^{\alpha(z)}-\omega}
$$

and thus

$$
f(z)+1=\omega\{g(z)+1\},
$$

in this case $a=2 \omega-1$ and $b=-2 \omega-3$. Next we suppose that $2 \alpha+\gamma=y$ is a constant, and so

$$
\begin{gathered}
2(a+1)(a-b) e^{-y} e^{\alpha}+4(a b+2 b-3) e^{-\alpha}-(a+3)\{(a-3)+b(a+1)\} e^{-2 \alpha} \\
=(b-1)\left\{4-(1+a)^{2} e^{-y}\right\},
\end{gathered}
$$

which implies similarly that the coefficients are zero. Then we see $a=b=-3$ by $a \neq \pm 1$ and $b \neq 1$, and also $e^{\beta}=e^{x}=1 / 2(a+1)=-1$ and $e^{y}=1$ or $e^{\gamma}=e^{-2 \alpha}$, so that

$$
f(z)=\frac{1-3 e^{\alpha(z)}}{e^{\alpha(z)}+1} \text { and } g(z)=\frac{e^{\alpha(z)}-3}{e^{\alpha(z)}+1}
$$


and thus

$$
f(z)+g(z)=-2 .
$$

From now on, we may regard $\beta$ as a nonconstant entire function.

Case (II): $\gamma=x$ is a constant.

By (5) we then have an identity

$$
\begin{aligned}
4 e^{2 \beta} & -2(a+b+2) e^{\beta}+2(a b-1)\left(e^{x}-1\right) e^{-\alpha+\beta}+2(a+b-2) e^{-\alpha+2 \beta}-2(a+b-2) e^{x} e^{-\alpha} \\
& +\left\{(1-a)(1-b)-(1+a)(1+b) e^{x}\right\} e^{-2 \alpha+2 \beta}+2(a+b+2) e^{x} e^{-2 \alpha+\beta}-4 e^{x} e^{-2 \alpha} \\
= & (1-a)(1-b) e^{x}-(1+a)(1+b) .
\end{aligned}
$$

For the case where one of $-\alpha+\beta,-\alpha+2 \beta$ and $-2 \alpha+\beta$ is a constant, (8) gives a contradiction by Lemma 1 , so that $(1-a)(1-b) e^{x}=(1+a)(1+b)$ and

$$
\begin{aligned}
& 2(1-a)(1-b) e^{2 \alpha+2 \beta}-(1-a)(1-b)(a+b+2) e^{2 \alpha+\beta}+2(a b-1)(a+b) e^{\alpha+\beta} \\
+ & (1-a)(1-b)(a+b-2) e^{\alpha+2 \beta}-(1+a)(1+b)(a+b-2) e^{\alpha}-2(a b+1)(a+b) e^{2 \beta} \\
+ & (1+a)(1+b)(a+b+2) e^{\beta}=2(1+a)(1+b) .
\end{aligned}
$$

Thus at least one of $\alpha+\beta, 2 \alpha+\beta$ and $\alpha+2 \beta$ must be a constant, however $a \neq 1$ and $b \neq 1$ deny the possibilities of $2 \alpha+\beta$ and $\alpha+2 \beta$ with a simple calculation. Suppose $\alpha+\beta$ to be a constant $y$ and so $\alpha=y-\beta$ is not. Identity (9) yields now

$$
\begin{aligned}
& \left\{(1-a)(1-b)(a+b+2) e^{y}+(1+a)(1+b)(a+b-2)\right\} e^{\alpha} \\
- & \left\{(1-a)(1-b)(a+b-2) e^{y}+(1+a)(1+b)(a+b+2)\right\} e^{y} e^{-\alpha}+2(a b+1)(a+b) e^{2 y} e^{-2 \alpha} \\
= & 2(1-a)(1-b) e^{2 y}+2(a b-1)(a+b) e^{y}-2(1+a)(1+b) .
\end{aligned}
$$

Here it follows that all the coefficients are equal to zero, and an elementary analysis shows that $a=-b, e^{y}=e^{\alpha+\beta}=1$ and $e^{\gamma}=e^{x}=(1+a)(1+b) /\{(1-a)(1-b)\}$ $=1$, therefore

$$
f(z)=\frac{1-e^{\alpha(z)}}{1+e^{\alpha(z)}} \text { and } \quad g(z)=\frac{e^{\alpha(z)}-1}{e^{\alpha(z)}+1},
$$

which are connected with

$$
f(z)=-g(z) .
$$

Hence we may assume that $\gamma$ is not a constant, either.

Case (III): $-\alpha+\beta=x$ is a constant with $e^{x} \neq 1$.

Then we note that $\alpha=\beta-x$ is not a constant and the starting identity is 


$$
\begin{aligned}
& 4 e^{2 x} e^{2 \alpha}+2 e^{x}\left\{(a+b-2) e^{x}-(a+b+2)\right\} e^{\alpha} \\
- & \left\{(1+a)(1+b) e^{2 x}-2(a b-1) e^{x}+(1-a)(1-b)\right\} e^{\gamma} \\
+ & 2\left\{(a+b+2) e^{x}-(a+b-2)\right\} e^{-\alpha+\gamma}-4 e^{-2 \alpha+\gamma} \\
= & -(1-a)(1-b) e^{2 x}+2(a b-1) e^{x}-(1+a)(1+b) .
\end{aligned}
$$

Here we suppose that the right-hand side is equal to zero. Then (10) gives

$$
\begin{aligned}
& 4 e^{2 x} e^{4 \alpha-\gamma}+2 e^{x}\left\{(a+b-2) e^{x}-(a+b+2)\right\} e^{3 \alpha-\gamma} \\
- & \left\{(1+a)(1+b) e^{2 x}-2(a b-1) e^{x}+(1-a)(1-b)\right\} e^{2 \alpha} \\
+ & 2\left\{(a+b+2) e^{x}-(a+b-2)\right\} e^{\alpha}=4 .
\end{aligned}
$$

Thus either $4 \alpha-\gamma$ or $3 \alpha-\gamma$ must be a constant $y$. If so is $4 \alpha-\gamma$,

$$
\begin{aligned}
& 2 e^{x}\left\{(a+b-2) e^{x}-(a+b+2)\right\} e^{y} e^{-\alpha} \\
- & \left\{(1+a)(1+b) e^{2 x}-2(a b-1) e^{x}+(1-a)(1-b)\right\} e^{2 \alpha} \\
+ & 2\left\{(a+b+2) e^{x}-(a+b-2)\right\} e^{\alpha}=4\left(1-e^{2 x+y}\right),
\end{aligned}
$$

whose consequence is the following; $e^{2 x+y}=1, e^{x}=-1$ and $a=b=0$, or $e^{\beta}=-e^{\alpha}$ and $e^{\gamma}=e^{4 \beta}$ so that

and

$$
f(z)=-e^{\alpha(z)} \text { and } \quad g(z)=-e^{-\alpha(z)}
$$

$$
f(z) \cdot g(z)=1 \text {. }
$$

While $3 \alpha-\gamma$ is a constant $y$,

$$
\begin{aligned}
& \left\{(1+a)(1+b) e^{2 x}-2(a b-1) e^{x}+(1-a)(1-b)\right\} e^{2 \alpha} \\
- & 2\left\{(a+b+2) e^{x}+2 e^{2 x+y}-(a+b-2)\right\} e^{\alpha}=2\left[e^{x+y}\left\{(a+b-2) e^{x}-(a+b+2)\right\}-2\right],
\end{aligned}
$$

which leads us to the consequence that $e^{y}=1, e^{2 x}+e^{x}+1=0, a+b=0$ and $a b=$ $1 / 3$. Then $e^{x}=1 / 2(-1 \pm \sqrt{3} i)=\omega$, say, and $a=-b= \pm i / \sqrt{3}, e^{\beta}=\omega e^{\alpha}, e^{\gamma}=e^{3 \alpha}$, so that in this case

$$
f(z)=\frac{2 \omega e^{\alpha(z)}-(1+\omega)}{1-\omega} \text { and } g(z)=\frac{-2 e^{-\alpha(z)}+(1+\omega)}{1-\omega}
$$

and therefore

$$
\left(f(z)+\frac{1+\omega}{1-\omega}\right)\left(g(z)-\frac{1+\omega}{1-\omega}\right)=\frac{4}{3} .
$$

Next we assume $(1-a)(1-b) e^{2 x}-2(a b-1) e^{x}+(1+a)(1+b) \neq 0$ in $(10)$. Then either $-\alpha+\gamma$ or $-2 \alpha+\gamma$ must be a constant. It is easy to show that the former is not. For the latter case we can find only one possibility that $e^{x}=-1$ and $e^{\gamma}=e^{2 \alpha}$, which gives the same as the previous for $4 \alpha-\gamma$, however in this case 
$a \cdot b=1$.

Now we suppose $-\alpha+\beta$ to be nonconstant.

Case $(I V):-\alpha+2 \beta=x$ is a constant.

Here $\alpha=2 \beta-x$ is not a constant and

$$
\begin{aligned}
& 4 e^{2 \beta}-2(a+b+2) e^{\beta}-(1-a)(1-b) e^{\gamma}-2(a b-1) e^{x} e^{-\beta}+2(a b-1) e^{x} e^{-\beta+\gamma} \\
+ & (1-a)(1-b) e^{2 x} e^{-2 \beta}-\left\{(1+a)(1+b) e^{x}+2(a+b-2)\right\} e^{x} e^{-2 \beta+\gamma} \\
+ & 2(a+b+2) e^{2 x} e^{-3 \beta+\gamma}-4 e^{2 x} e^{-4 \beta+\gamma}=-2(a+b-2) e^{x}-(1+a)(1+b) .
\end{aligned}
$$

In this case, it is only the case sifted out that $-2 \beta+\gamma=y$ is a constant with $2(a+b-2) e^{x}+(1+a)(1+b) \neq 0$, and the consequence is that $e^{x}=1, e^{y}=1$ or $e^{\alpha}=$ $e^{2 \beta}=e^{r}$ with $(1-a)(1-b)=4$. Thus

$$
f(z)=2 e^{\beta(z)}+1 \text { and } g(z)=2 e^{-\beta(z)}+1
$$

and they posess the relationship

$$
\{f(z)-1\}\{g(z)-1\}=4 .
$$

Case $(V):-\alpha+\beta+\gamma=x$ is a constant.

An identity

$$
\begin{aligned}
& -(1-a)(1-b) e^{x} e^{\alpha-\beta}+4 e^{2 \beta}-2(a+b+2) e^{\beta}-2(a+b-2) e^{x} e^{-\beta}+2(a+b+2) e^{x} e^{-\alpha} \\
& +2(a+b-2) e^{-\alpha+2 \beta}-\left\{(1+a)(1+b) e^{x}+2(a b-1)\right\} e^{-\alpha+\beta}-4 e^{x} e^{-\alpha-\beta} \\
& +(1-a)(1-b) e^{-2 \alpha+2 \beta}=-2(a b-1) e^{x}-(1+a)(1+b)
\end{aligned}
$$

permits us only to imply that $\alpha=y$ is a constant with $2(a b-1) e^{x}+(1+a)(1+b) \neq 0$ and then $e^{y}=1 / 2(-1 \pm \sqrt{3} i)=\omega, e^{x}=\omega^{2}$, that is, $e^{\alpha}=\omega, e^{\gamma}=e^{-\beta}$, and

satisfied by

$$
f(z)-1=\omega\{g(z)-1\}
$$

$$
f(z)=\frac{(2 \omega-1) e^{\beta(z)}-\omega}{\omega-e^{\beta(z)}} \text { and } g(z)=\frac{e^{\beta(z)}+(\omega-2)}{\omega-e^{\beta(z)}},
$$

with $\{a, b\}=\{2 \omega+3,1-2 \omega\}$.

Case $(V I):-2 \alpha+2 \beta+\gamma=x$ is a constant.

Then the identity (5) induces us to get 


$$
\begin{aligned}
& -(1-a)(1-b) e^{x} e^{2 \alpha-2 \beta}+2(a b-1) e^{x} e^{\alpha-\beta}-2(a+b-2) e^{x} e^{\alpha-2 \beta}+4 e^{2 \beta}-2(a+b+2) e^{\beta} \\
& +2(a+b+2) e^{x} e^{-\beta}-4 e^{x} e^{-2 \beta}+2(a+b-2) e^{-\alpha+2 \beta}-2(a b-1) e^{-\alpha+\beta} \\
& +(1-a)(1-b) e^{-2 \alpha+2 \beta}=(1+a)(1+b)\left(e^{x}-1\right) .
\end{aligned}
$$

We know that all functions in this left-hand side are nonconstant, therefore the right-hand side, that is $e^{x}-1$, must be equal to zero. Then we have a further identity which shows that $\alpha=y$ must be a constant with $e^{y} \neq 1$, and thus

$$
\begin{aligned}
& 2\left\{(a b-1) e^{y}+(a+b+2)\right\} e^{\beta}-2\left\{(a b-1) e^{-y}+(a+b+2)\right\} e^{3 \beta} \\
+ & \left\{(1-a)(1-b) e^{-2 y}+2(a+b-2) e^{-y}+4\right\} e^{4 \beta}=(1-a)(1-b) e^{y}+2(a+b-2) e^{y}+4 .
\end{aligned}
$$

Since $\beta$ is not a constant, all the coefficients must be zero and so we derive $e^{y}=-1$ and $a=b=3$, or $e^{\alpha}=-1$ and $e^{\gamma}=e^{-2 \beta}$, thus

$$
f(z)=\frac{3 e^{\beta(z)}-1}{1+e^{\beta(z)}} \text { and } g(z)=\frac{3-e^{\beta(z)}}{1+e^{\beta(z)}},
$$

which gives a relationship

$$
f(z)+g(z)=2 .
$$

Case $(V I I):-2 \alpha+\gamma=x$ is a constant.

This is the last case that bears the fruitful consequence. It follows from the identity

$$
\begin{aligned}
& -(1-a)(1-b) e^{x} e^{2 \alpha}+2(a b-1) e^{x} e^{\alpha+\beta}-2(a+b-2) e^{x} e^{\alpha}+\left\{4-(1+a)(1+b) e^{x}\right\} e^{2 \beta} \\
& +2(a+b+2)\left(e^{x}-1\right) e^{\beta}+2(a+b-2) e^{-\alpha+2 \beta}-2(a b-1) e^{-\alpha+\beta}+(1-a)(1-b) e^{-2 \alpha+2 \beta} \\
= & 4 e^{x}-(1+a)(1+b)
\end{aligned}
$$

together with the assumption that $-2 \alpha+\beta$ is a constant $y$,

$$
\begin{aligned}
& \left\{16-(1+a)^{2}(1+b)^{2}\right\} e^{2 y} e^{2 \alpha}+2 e^{y}\left\{4(a+b-2) e^{y}+(1+a)(1+b)(a b-1)\right\} e^{\alpha} \\
- & 2\left\{4(a b-1) e^{y}+(1+a)(1+b)(a+b-2)\right\} e^{-\alpha} \\
= & -4(1-a)(1-b) e^{2 y}-2(a+b+2)\{(1+a)(1+b)-4\} e^{y}+\left(1-a^{2}\right)\left(1-b^{2}\right) .
\end{aligned}
$$

Hence all the coefficients must be zero, therefore we derive that $(1+a)(1+b)=4$, and $e^{x}=e^{y}=1$, or $e^{\beta}=e^{2 \alpha}=e^{\gamma}$. Then

$$
f(z)=-2 e^{\alpha(z)}-1 \text { and } g(z)=-2 e^{-\alpha(z)}-1
$$

and they are connected with the relationship

$$
\{f(z)+1\}\{g(z)+1\}=4 \text {. }
$$

Like this manner the last possibility has been found in our theorem. 


\section{Proof of Theorem 5 .}

According to the assumptions, following four equations are obtained.

and

$$
\left\{\begin{array}{l}
\left(g-a_{1}\right)\left(g-a_{2}\right)=e^{\alpha}\left(f-a_{1}\right)\left(f-a_{2}\right) \\
\left(g-b_{1}\right)\left(g-b_{2}\right)=e^{\beta}\left(f-b_{1}\right)\left(f-b_{2}\right)
\end{array}\right.
$$

$$
\left\{\begin{array}{l}
\left(h-a_{1}\right)\left(h-a_{2}\right)=e^{r}\left(f-a_{1}\right)\left(f-a_{2}\right) \\
\left(h-c_{1}\right)\left(h-c_{2}\right)=e^{\delta}\left(f-c_{1}\right)\left(f-c_{2}\right)
\end{array}\right.
$$

where $\alpha, \beta, \gamma$ and $\delta$ are entire functions. Each difference of these two pairs (1), (2) yields

$$
\begin{aligned}
& a_{1} a_{2}-b_{1} b_{2}=\left(e^{\alpha}-e^{\beta}\right) f^{2}+d\left(e^{\beta}-e^{\alpha}\right) f+a_{1} a_{2} e^{\alpha}-b_{1} b_{2} e^{\beta}, \\
& a_{1} a_{2}-c_{1} c_{2}=\left(e^{\gamma}-e^{\delta}\right) f^{2}+d\left(e^{\delta}-e^{\gamma}\right) f+a_{1} a_{2} e^{\gamma}-c_{1} c_{2} e^{\delta} .
\end{aligned}
$$

Since $f$ is not a constant, we may assume that either of $\alpha$ and $\beta$ is not a constant and neither is $\gamma$ nor $\delta$. Moreover we may suppose that both $e^{\alpha}-e^{\beta}$ and $e^{\gamma}-e^{\delta}$ are not identically equal to zero, otherwise (3) or (4) shows $e^{\alpha}=e^{\beta} \equiv 1$ or $e^{\gamma}=e^{\delta} \equiv 1$ so that one of $f=g, f+g=d, f=h$ and $f+h=d$. Then it follows as well as $g$ and $h$,

$$
\begin{aligned}
f & =\frac{d}{2} \pm\left[\frac{d^{2}}{4}-\frac{a_{1} a_{2}\left(e^{\alpha}-1\right)-b_{1} b_{2}\left(e^{\beta}-1\right)}{e^{\alpha}-e^{\beta}}\right]^{1 / 2} \\
& =\frac{d}{2} \pm\left[\frac{d^{2}}{4}-\frac{a_{1} a_{2}\left(e^{\gamma}-1\right)-c_{1} c_{2}\left(e^{\delta}-1\right)}{e^{\gamma}-e^{\delta}}\right]^{1 / 2},
\end{aligned}
$$

and so

$$
B e^{\alpha-\delta}-B e^{\alpha}-B e^{\beta-\delta}+C e^{\beta+\gamma-\delta}+A e^{\beta}-C e^{\gamma-\delta}=-C,
$$

setting $A=b_{1} b_{2}-c_{1} c_{2}, B=a_{1} a_{2}-c_{1} c_{2}$ and $C=a_{1} a_{2}-b_{1} b_{2}$, which are all different from zero.

In this proof we shall see the possible combinations of $\alpha, \beta, \gamma$ and $\delta$ only. Then we note that the resulted representations for $f, g$ and $h$ need to be screened under the criterion that a function in a square root never has a simple zero and a simple pole since our functions are meromorphic.

Case (i): $\alpha-\delta=x,-2 \alpha+\beta+\gamma=y$ and $-\alpha+\beta=z$ are all constants with $B e^{x}+C=0$ and $C^{2} e^{y}+B^{2} \neq 0$.

Then (6) gives $C\left(B e^{2 z}+C e^{y}\right) e^{-z} e^{-\alpha}=C^{2} e^{y}-A B e^{z}+B^{2}$, so that both $B e^{2 z}+C e^{y}$ and $C^{2} e^{y}-A B e^{z}+B^{2}$ are equal to zero, since $\alpha=\beta-z$ must be nonconstant as mentioned above. Thus we derive (i) $e^{z}=\{-A \pm(B+C)\} /(2 C)=1,-B / C$ if $B+C \neq 0$, (ii) $e^{z}=A /(2 B)$ if $B+C=0$. For (i), $e^{z}=1$ is omitted by $e^{\alpha}-e^{\beta} \neq 0$, 
while $e^{z}=-B / C$ gives $e^{y}=-(B / C)^{3}$ and now $e^{x}=-C / B$, so that $e^{\beta}=-(B / C) e^{\alpha}$, $e^{\gamma}=(B / C)^{2} e^{\alpha}$ and $e^{\delta}=-(B / C) e^{\alpha}$, by which (5) implies

$$
\begin{aligned}
f & =\frac{d}{2} \pm\left[\left(\frac{d^{2}}{4}-\frac{a_{1} a_{2} C+b_{1} b_{2} B}{B+C}\right)+\frac{C}{1+B / C} e^{-\alpha}\right]^{1 / 2} \\
& =\frac{d}{2} \pm\left[\left(\frac{d^{2}}{4}-\frac{a_{1} a_{2} B+c_{1} c_{2} C}{B+C}\right)+\frac{B}{(B+C) B / C^{2}} e^{-\alpha}\right]^{1 / 2}
\end{aligned}
$$

and also

$$
\begin{aligned}
& g=\frac{d}{2} \pm\left[\left(\frac{d^{2}}{4}-\frac{a_{1} a_{2} B+b_{1} b_{2} C}{B+C}\right)+\frac{C}{1+C / B} e^{\alpha}\right]^{1 / 2}, \\
& h=\frac{d}{2} \pm\left[\left(\frac{d^{2}}{4}-\frac{a_{1} a_{2} C+c_{1} c_{2} B}{B+C}\right)+\frac{B}{(B+C) C / B^{2}} e^{\alpha}\right]^{1 / 2} .
\end{aligned}
$$

By the reason mentioned above, it must be satisfied that

$$
\left\{\begin{array} { l } 
{ \frac { d ^ { 2 } } { 4 } - \frac { a _ { 1 } a _ { 2 } C + b _ { 1 } b _ { 2 } B } { B + C } = 0 } \\
{ \frac { d ^ { 2 } } { 4 } - \frac { a _ { 1 } a _ { 2 } B + b _ { 1 } b _ { 2 } C } { B + C } = 0 }
\end{array} \text { and } \left\{\begin{array}{l}
\frac{d^{2}}{4}-\frac{a_{1} a_{2} B+c_{1} c_{2} C}{B+C}=0 \\
\frac{d^{2}}{4}-\frac{a_{1} a_{2} C+c_{1} c_{2} B}{B+C}=0,
\end{array}\right.\right.
$$

which implies, however, $B=C$ or $b_{1} b_{2}=c_{1} c_{2}$. This is a contradiction.

With respect to (ii), $e^{z}=A /(2 B), e^{y}=e^{2 z}$ and $e^{x}=1$ so that $e^{\beta}=1 / 2(A / B) e^{\alpha}$, $e^{\gamma}=1 / 2(A / B) e^{\alpha}$ and $e^{\delta}=e^{\alpha}$. Here we note $A \neq 2 B$ by $e^{z}=e^{-\alpha+\beta} \neq 1$. Then

$$
\begin{aligned}
f & =\frac{d}{2} \pm\left[\left(\frac{d^{2}}{4}-\frac{2 a_{1} a_{2} B-b_{1} b_{2} A}{2 B-A}\right)+\frac{2 B C}{2 B-A} e^{-\alpha}\right]^{1 / 2} \\
& =\frac{d}{2} \pm\left[\left(\frac{d^{2}}{4}-\frac{a_{1} a_{2} A-2 c_{1} c_{2} B}{A-2 B}\right)+\frac{2 B^{2}}{2 B-A} e^{-\alpha}\right]^{1 / 2}, \\
g & =\frac{d}{2} \pm\left[\left(\frac{d^{2}}{4}-\frac{a_{1} a_{2} A-2 b_{1} b_{2} B}{A-2 B}\right)+\frac{A C}{A-2 B} e^{\alpha}\right]^{1 / 2}, \\
h & =\frac{d}{2} \pm\left[\left(\frac{d^{2}}{4}-\frac{2 a_{1} a_{2} B-c_{1} c_{2} A}{2 B-A}\right)+\frac{A C}{2 B-A} e^{\alpha}\right]^{1 / 2},
\end{aligned}
$$

and the same observation as (i) leads us to $A+2 B=A+B-C=2 A=0$. Hence there are no desired meromorphic functions in this case.

Case (ii): $\quad \alpha-\delta=x,-\alpha+\beta=y$ and $-\alpha+\gamma=z$ are constants.

In the present case, (6) gives $\left(A e^{y}+C e^{x+y+z}-B\right) e^{\alpha}=B e^{x}\left(e^{y}-1\right)+C\left(e^{x+z}-1\right)$. Since $\alpha=\beta-y$ is not a constant, it follows that

$$
A e^{y}+C e^{x+y+z}-B=0 \text { and } B e^{x}\left(e^{y}-1\right)+C\left(e^{x+z}-1\right)=0 \text {, }
$$

and so we derive either (i) $e^{y}=1$ and $e^{x+z}=1$, or (ii) $e^{x+y}=1$ and $A e^{y}+C e^{z}=B$. 
The former (i) is however the omitted case in which $e^{\alpha}=e^{\beta}$ and $e^{\gamma}=e^{\delta}$. For the remainder (ii), it may be assumed since now that $e^{y} \neq 1$ and $e^{z} \neq e^{y}$, and $e^{\beta}=$ $e^{y} e^{\alpha}, e^{\gamma}=e^{z} e^{\alpha}, e^{\delta}=e^{\beta}$. From them

$$
\begin{aligned}
f & =\frac{d}{2} \pm\left[\left(\frac{d^{2}}{4}-\frac{a_{1} a_{2}-b_{1} b_{2} e^{y}}{1-e^{y}}\right)+\frac{C}{1-e^{y}} e^{-\alpha}\right]^{1 / 2} \\
& =\frac{d}{2} \pm\left[\left(\frac{d^{2}}{4}-\frac{a_{1} a_{2} e^{z}-c_{1} c_{2} e^{y}}{e^{z}-e^{y}}\right)+\frac{B}{e^{z}-e^{y}} e^{-\alpha}\right]^{1 / 2}, \\
g & =\frac{d}{2} \pm\left[\left(\frac{d^{2}}{4}-\frac{a_{1} a_{2}-b_{1} b_{2} e^{-y}}{1-e^{-y}}\right)+\frac{C}{1-e^{-y}} e^{\alpha}\right]^{1 / 2}, \\
h & =\frac{d}{2} \pm\left[\left(\frac{d^{2}}{4}-\frac{a_{1} a_{2} e^{-z}-c_{1} c_{2} e^{-y}}{e^{-z}-e^{-y}}\right)+\frac{B}{e^{-z}-e^{-y}} e^{\alpha}\right]^{1 / 2},
\end{aligned}
$$

and the observation gives the conclusion $e^{z}=-e^{y}=1$ so that it follows from $A e^{y}+C e^{z}=B$, a contradiction $-A-B+C=-2 A=0$.

Case (iii): $\alpha-\delta=x,-\alpha+\gamma=y$ are constants.

Then the starting identity is

$$
B e^{\alpha}+B e^{x} e^{-\alpha+\beta}-\left(A+C e^{x+y}\right) e^{\beta}=B e^{x}-C e^{x+y}+C .
$$

We can make it sure that $\alpha,-\alpha+\beta$ and $\beta$ are all nonconstant in the usual way and so $B e^{x}-C e^{x+y}+C=0$ and $B e^{\alpha-\beta}+B e^{x} e^{-\alpha}=C e^{x+y}+A$, which implies $e^{2 \alpha}=-e^{x} e^{\beta}, e^{x}=-1$ and $e^{y}=A / C$, or $e^{\beta}=e^{2 \alpha}, e^{r}=(A / C) e^{\alpha}$ and $e^{\delta}=-e^{\alpha}$. Thus

$$
\begin{aligned}
f & =\frac{d}{2} \pm\left[\left(\frac{d^{2}}{4}-b_{1} b_{2}\right)+C e^{-\alpha}\right]^{1 / 2} \\
& =\frac{d}{2} \pm\left[\left(\frac{d^{2}}{4}-\frac{a_{1} a_{2} A+c_{1} c_{2} C}{A+C}\right)+\frac{A B}{A+C} e^{-\alpha}\right]^{1 / 2}, \\
g & =\frac{d}{2} \pm\left[\left(\frac{d^{2}}{4}-b_{1} b_{2}\right)+C e^{\alpha}\right]^{1 / 2}, \\
h & =\frac{d}{2} \pm\left[\left(\frac{d^{2}}{4}-\frac{a_{1} a_{2} C+c_{1} c_{2} A}{A+C}\right)+\frac{A B}{A+C} e^{\alpha}\right]^{1 / 2},
\end{aligned}
$$

so that we derive $d^{2} / 4-b_{1} b_{2}=1 / 4\left(b_{1}-b_{2}\right)^{2}=0$ or $b_{1}=b_{2}$, and $A=C$ or $a_{1} a_{2}-c_{1} c_{2}$ $=2\left(b_{1} b_{2}-c_{1} c_{2}\right)$, then

$$
\begin{aligned}
& f=\frac{d}{2} \pm\left(C e^{-\alpha}\right)^{1 / 2}=\frac{d}{2} \pm\left(\frac{1}{2} B e^{-\alpha}\right)^{1 / 2}, \\
& g=\frac{d}{2} \pm\left(C e^{\alpha}\right)^{1 / 2}, \quad h=\frac{d}{2} \pm\left(\frac{1}{2} B e^{\alpha}\right)^{1 / 2} .
\end{aligned}
$$

They are the desired functions given in (III). 
Case (iv): $\alpha=x, \beta+\delta=y$ and $\gamma=z$ are constants with $e^{x}=C / B$ and $e^{y} \neq-C / A$.

By (6) we have $\left(C e^{z}-B\right) e^{\beta}=C e^{z}-A e^{y}-C$ and thus $C e^{z}-B=C e^{z}-A e^{y}-C=0$, since $\beta$ must be nonconstant now. The consequence is that $e^{x}=e^{-z}=C / B$ and $e^{y}=1$, or $e^{\gamma}=e^{-\alpha}=B / C$ and $e^{\delta}=e^{-\beta}$. Then we have

$$
\begin{aligned}
f & =\frac{d}{2} \pm \frac{1}{2}\left[\frac{\left(b_{1}-b_{2}\right)^{2} e^{\beta}-\left(a_{1}-a_{2}\right)^{2} C / B-4 C}{e^{\beta}-C / B}\right]^{1 / 2} \\
& =\frac{d}{2} \pm \frac{1}{2}\left[\frac{\left(c_{1}-c_{2}\right)^{2} e^{-\beta}-\left(a_{1}-a_{2}\right)^{2} B / C-4 B}{e^{-\beta}-B / C}\right]^{1 / 2}, \\
g & =\frac{d}{2} \pm \frac{1}{2}\left[\frac{\left(b_{1}-b_{2}\right)^{2} e^{-\beta}-\left(a_{1}-a_{2}\right)^{2} B / C-4 C}{e^{-\beta}-B / C}\right]^{1 / 2}, \\
h & =\frac{d}{2} \pm \frac{1}{2}\left[\frac{\left(c_{1}-c_{2}\right)^{2} e^{\beta}-\left(a_{1}-a_{2}\right)^{2} C / B-4 B}{e^{\beta}-C / B}\right]^{1 / 2} .
\end{aligned}
$$

Now each function in a square root cannot have any simple pole and zero, so that its denominator and numerator share $0 C M$, according to their forms. To do this, we need $C(C-B)=0$, which is however impossible.

Case $(v): \quad \beta-\delta=x,-\alpha+2 \beta=y$ and $-2 \beta+\gamma=z$ are constants with $e^{x}=C / B$.

Then (6) is of the form $\left(B^{2}-C^{2} e^{y+z}\right) e^{\beta}=A B e^{y}-C^{2} e^{y+z}+B C$ whose consequence is that $e^{y}=1$ and $e^{z}=B^{2} / C^{2}$, thus $e^{\alpha}=e^{2 \beta}, e^{\gamma}=\left(B^{2} / C^{2}\right) e^{2 \beta}$ and $e^{\delta}=$ $(B / C) e^{\beta}$. The representations for $f, g$ and $h$ follow from them, that is,

$$
\begin{aligned}
& f=\frac{d}{2} \pm\left[\frac{1}{4}\left(a_{1}-a_{2}\right)^{2}-C e^{-\beta}\right]^{1 / 2}=\frac{d}{2} \pm\left[\frac{1}{4}\left(a_{1}-a_{2}\right)^{2}-\frac{B}{B / C} e^{-\beta}\right]^{1 / 2}, \\
& g=\frac{d}{2} \pm\left[\frac{1}{4}\left(a_{1}-a_{2}\right)^{2}-C e^{\beta}\right]^{1 / 2}, \quad h=\frac{d}{2} \pm\left[\frac{1}{4}\left(a_{1}-a_{2}\right)^{2}-\frac{B}{C / B} e^{\beta}\right]^{1 / 2} .
\end{aligned}
$$

Hence by $a_{1}=a_{2}$ and setting $K=B / C \neq 0,1$, we obtain the result (IV).

Case (vi): $\beta-\delta=x, \beta=y$ are constants.

In this case by (6)

$$
B\left(e^{x-y}-1\right) e^{\alpha}+C e^{x-y}\left(e^{y}-1\right) e^{\gamma}=B e^{x}-A e^{y}-C .
$$

Since $\alpha$ and $\gamma$ must be nonconstant, this gives $B e^{x}-A e^{y}=C$ and $B\left(e^{x-y}-1\right) e^{\alpha-r}$ $=C e^{x-y}\left(1-e^{y}\right)$. If $e^{x-y}=1, e^{x}=e^{y}=1$ or $e^{\beta}=e^{\delta} \equiv 1$, which is reduced to the result (I). Otherwise we obtain $e^{\gamma}=e^{-x} e^{\alpha}$ in consideration of $B e^{x}-A e^{y}=C$. Then it follows by $e^{\beta} \equiv e^{y}$ and $e^{\delta} \equiv e^{-x+y}$ with $B e^{x}=A e^{y}+C$, the representation:

$$
f=\frac{d}{2} \pm \frac{1}{2}\left[\frac{\left(a_{1}-a_{2}\right)^{2} e^{\alpha}-\left(b_{1}-b_{2}\right)^{2} e^{y}+4 C}{e^{\alpha}-e^{y}}\right]^{1 / 2}
$$




$$
=\frac{d}{2} \pm \frac{1}{2}\left[\frac{\left(a_{1}-a_{2}\right)^{2} e^{\alpha}-\left(c_{1}-c_{2}\right)^{2} e^{y}+4 B e^{x}}{e^{\alpha}-e^{y}}\right]^{1 / 2} .
$$

Those for $g$ and $h$ are obtained by replacing $\alpha$ by $-\alpha$ and similarly for $x$ and $y$. The observation implies $e^{x}=e^{y}=1$, which is omitted above.

Case (vii): $\beta+\gamma-\delta=x, \alpha-\gamma=y$ and $\alpha+\beta=z$ are constants with $e^{x}=-1$.

Then (6) gives us an identity $e^{z}\left(A e^{z}+B e^{y}\right) e^{-2 \alpha}=B e^{z}+B e^{y}-C$, which leads further to the result that $e^{z}=1$ and $e^{y}=-A / B$ since $\alpha=-\beta+z$ is not a constant. Thus $e^{\beta}=e^{-\alpha}, e^{\gamma}=-(B / A) e^{\alpha}$ and $e^{\delta}=B / A$, so that

$$
\begin{aligned}
f & =\frac{d}{2} \pm \frac{1}{2}\left[\frac{\left(a_{1}-a_{2}\right)^{2} e^{2 \alpha}+4 C e^{\alpha}-\left(b_{1}-b_{2}\right)^{2}}{e^{2 \alpha}-1}\right]^{1 / 2} \\
& =\frac{d}{2} \pm \frac{1}{2}\left[\frac{\left(a_{1}-a_{2}\right)^{2} e^{\alpha}+\left(c_{1}-c_{2}\right)^{2}-A}{e^{\alpha}+1}\right]^{1 / 2}, \\
g & =\frac{d}{2} \pm \frac{1}{2}\left[\frac{\left(b_{1}-b_{2}\right)^{2} e^{2 \alpha}-4 C e^{\alpha}-\left(a_{1}-a_{2}\right)^{2}}{e^{2 \alpha}-1}\right]^{1 / 2}, \\
h & =\frac{d}{2} \pm \frac{1}{2}\left[\frac{\left(a_{1}-a_{2}\right)^{2} e^{-\alpha}+\left(c_{1}-c_{2}\right)^{2}-B^{2} / A}{e^{-\alpha}+1}\right]^{1 / 2} .
\end{aligned}
$$

It thus follows that either $B$ or $C$ must be equal to zero, which is impossible.

Case (viii): $\beta+\gamma-\delta=x, \alpha-\gamma=y$ and $-\alpha+\beta=z$ are constants with $e^{x}=-1$.

Then $e^{z}\left(A e^{z}-B\right) e^{2 \alpha}=B e^{y}-B e^{y+z}-C$ and thus $e^{z}=B / A$, $e^{y}=-A / B$, so that we obtain $e^{\beta}=(B / A) e^{\alpha}, e^{\gamma}=-(B / A) e^{\alpha}, e^{\delta}=(B / A)^{2} e^{2 \alpha}$ and the representation ;

$$
\begin{aligned}
f & =\frac{d}{2} \pm\left[\left(\frac{d^{2}}{4}-\frac{a_{1} a_{2} A-b_{1} b_{2} B}{A-B}\right)+\frac{A C}{A-B} e^{-\alpha}\right]^{1 / 2} \\
& =\frac{d}{2} \pm\left[\left(c_{1}-c_{2}\right)^{2}-A e^{-\alpha}\right]^{1 / 2}, \\
g & =\frac{d}{2} \pm\left[\left(\frac{d^{2}}{4}-\frac{a_{1} a_{2} B-b_{1} b_{2} A}{B-A}\right)+\frac{B C}{B-A} e^{\alpha}\right]^{1 / 2}, \\
h & =\frac{d}{2} \pm\left[\left(c_{1}-c_{2}\right)^{2}-\frac{B^{2}}{A} e^{\alpha}\right]^{1 / 2} .
\end{aligned}
$$

Hence we need $c_{1}=c_{2}$ and $a_{1} a_{2}-b_{1} b_{2}=2\left(a_{1} a_{2}-c_{1} c_{2}\right)$, then

$$
\begin{aligned}
& f=\frac{d}{2} \pm\left(\frac{1}{2} C e^{-\alpha}\right)^{1 / 2}=\frac{d}{2} \pm\left(B e^{-\alpha}\right)^{1 / 2}, \\
& g=\frac{d}{2} \pm\left(\frac{1}{2} C e^{\alpha}\right)^{1 / 2}, \quad h=\frac{d}{2} \pm\left(B e^{\alpha}\right)^{1 / 2},
\end{aligned}
$$


so that $g=h$ or $g+h=d$, which is found in (II).

Case (ix): $\beta=x$ and $\alpha+\delta=y$ are constants with $e^{x}=-C / A$.

This is the last case that we need to discuss, and now we have an identity

$$
B e^{\alpha}+C\left(e^{x}-1\right) e^{\gamma}=B\left(e^{x}+e^{y}\right),
$$

and thus $e^{x}=-e^{y}$ and $e^{\alpha-\gamma}=C / A$ together with $e^{x}=-C / A$. They imply the functions

$$
\begin{aligned}
f & =\frac{d}{2} \pm \frac{1}{2}\left[\frac{\left(a_{1}-a_{2}\right)^{2} e^{\alpha}+\left(b_{1}-b_{2}\right)^{2}(A / C)+4 C}{e^{\alpha}+C / A}\right]^{1 / 2} \\
& =\frac{d}{2} \pm \frac{1}{2}\left[\frac{\left(a_{1}-a_{2}\right)^{2}(A / C)^{2} e^{2 \alpha}+4(A B / C) e^{\alpha}-\left(c_{1}-c_{2}\right)^{2}}{(A / C)^{2} e^{2 \alpha}-1}\right]^{1 / 2}, \\
g & =\frac{d}{2} \pm \frac{1}{2}\left[\frac{\left(a_{1}-a_{2}\right)^{2} e^{-\alpha}+\left(b_{1}-b_{2}\right)^{2}(A / C)+4 C}{e^{-\alpha}+A / C}\right]^{1 / 2}, \\
h & =\frac{d}{2} \pm \frac{1}{2}\left[\frac{\left(a_{1}-a_{2}\right)^{2}(C / A)^{2} e^{-2 \alpha}+4(B C / A) e^{-\alpha}-\left(c_{1}-c_{2}\right)^{2}}{(C / A)^{2} e^{-2 \alpha}-1}\right]^{1 / 2},
\end{aligned}
$$

and so $A+C=B=0$ is needed in order to make these three meromorphic, and this is impossible.

These are only the case worth introducing here. We note that resulted three functions $f, g, h$, but (I) are entire and of a form $e^{L}+(1 / 2) d$ with an entire function $L$.

\section{REFERENCES}

[1] Gross, F., On the distribution of values of meromorphic functions, Trans. Amer. Math. Soc., 131 (1968), 199-214.

[2] Gross, F. AND C.F. OsGood, Entire functions with common preimages, Factorization theory of meromorphic functions, 19-24, Marcel Dekker, Inc., 1982.

[3] Gross, F. AND C.C. YANG, Meromorphic functions covering certain finite sets at the same points, Illinois J. Math., 26 (1982), 432-441.

[4] Gundersen, G.G., Meromorphic functions that share three or four values, J. London Math. Soc., (2), ?.0 (1979), 457-466.

[5] Hayman, W.K., Meromol phic Functions (Clarendon Press, Oxford, 1964).

[6] Hiromi, G. AND M. OzAwA, On the existence of analytic mappings between two ultrahyperelliptic surfaces, Kodai Math. Sem. Rep., 17 (1965), 281-306.

[7] MuEs, E., Über die Nullstellen homogener Differentialpolynome, manuscripta math., 23 (1978), 325-341.

[8] Nevanlinna, R., Einige Eindeutigekeitssätze in der Theorie der Meromorphen Funktionen, Acta. Math., 48 (1926), 367-391.

[9] Nevanlinna, R., Le théorème de Picard-Borel et la théorie des fonctions méromorphes (Gauthier-Villars, Paris, 1929).

[10] Ninno, K. And M. Ozawa, Deficiencies of an entire algebroid function, Kodai 
Math. Sem. Rep., 22 (1970), 98-113.

[11] OzAwA, M., On the zero-one set of an entire function II, Kodai Math. J., 2 (1979), 194-199.

[12] Rubel, L. A. ANd C. C. YANG, On zero-one sets for entire functions, Mich. Math. J., 20 (1973), 289-296.

[13] YANG, C.C., On two entire functions which together with their first derivatives has the same zeros, J. Math. Anal. Appl., 56 (1976), 1-6.

Department of Mathematics Science University of Tokyo Noda, Chiba, Japan 